\title{
Händels Solomon in der Bearbeitung von Felix Mendelssohn Bartholdy (1835)*
}

\author{
„[...] ich muß sagen, daß ich seitdem erst weiß, \\ wie man Händelsche Sachen aufführen soll.“"1
}

Von Mendelssohns aufführungspraktischer Einrichtung des Händel'schen Oratoriums Solomon, die am 7. Juni 1835 im Kölner Gürzenichsaal während des 17. Niederrheinischen Musikfestes unter Mitwirkung von über 600 Personen erstmals aufgeführt wurde, ${ }^{2}$ war bislang lediglich eine in der Musikabteilung der Staatsbibliothek zu Berlin verwahrte, autographe Orgelstimme zugänglich, ${ }^{3}$ die in diesem Beitrag als Berliner Stimme oder Berliner Autograph bezeichnet wird. ${ }^{4}$ Für das Musikfest erschien auch ein Programmheft mit einer deutschen Übersetzung des Oratorientextes im Druck. ${ }^{5}$ In der Bodleian Library der University of Oxford findet sich eine von Mendelssohn verfasste Auflistung der Oratoriensätze, ${ }^{6}$ die seine Auseinandersetzung mit Händels Werk erkennen lässt. Aus den dort genannten Seitenzahlen geht hervor, dass ihm die gedruckte Ausgabe Samuel Arnolds [ca. 1790] als Vorlage diente. ${ }^{7}$ Im Juli 1829 besuchte er die King's Library, die sich heute im St. Pancras Building der British Library (London) befindet, und fertigte ein „Register von Originalmanuscripten "8 dort vorhandener Händel'scher Oratorien an, das auch Salomon verzeichnet. ${ }^{9}$

* Der Verfasser möchte allen Bibliotheksangestellten der Hochschule für Musik und Tanz Köln, insbesondere Herrn Markus Ecker, den Mitarbeitern des Mendelssohn-Archivs der Staatsbibliothek zu Berlin und des Heinrich-Heine-Instituts Düsseldorf sowie folgenden Personen für ihre bereitwillige Hilfe und Unterstützung danken: Dirk Gehring, Bernhard Haas, Anselm Hartinger, Manfred Hößl, Arnold Jacobshagen, Erich Reimer, Heiner Rekeszus, Johannes Schild, Christian Stähr, Ralf Wehner.

1 Brief Mendelssohns vom 26.06.1835 an Carl Klingemann, in: Felix Mendelssohn Bartholdy, Sämtliche Briefe, Bd. 4, hrsg. von Lucian Schiwietz und Sebastian Schmideler, Kassel u. a. 2011, S. 255.

2 Vgl. Klaus Wolfgang Niemöller, „Felix Mendelssohn-Bartholdy und das Niederrheinische Musikfest 1835 in Köln“, in: Studien zur Musikgeschichte des Rheinlandes 3, hrsg. von Ursula Eckart-Bäcker (= Beiträge zur Rheinischen Musikgeschichte 62), Köln 1965, S. 46-64.

3 Vgl. Ralf Wehner, Felix Mendelssohn Bartholdy. Thematisch-systematisches Verzeichnis der musikalischen Werke $(M W V)$ (= Leipziger Ausgabe der Werke von Felix Mendelssohn Bartholdy [LMA] XIII/1A), Wiesbaden 2009, S. 506.

4 D-B, Musikabteilung mit Mendelssohn-Archiv, Mus. ms. autogr. F. Mendelssohn Bartholdy F. 28, S. 261-276.

5 „Text der Gesangstücke, welche beim Musikfeste in Köln am 7. und 8. Juni 1835 ausgeführt werden. Nebst einem Verzeichniß sämmtlicher Mitwirkenden“, Köln [1835], D-KNu RHK 1283-17.

6 GB-Ob M.D.M.d.30/211.

7 Solomon, A Sacred Oratorio In Score, With all the additional Alterations, Composed in the Year, 1749, By G. F. Handel, hrsg. von Samuel Arnold (= Arnold's edition 85-92), London [ca. 1790]. Beispielsweise bezieht sich Mendelssohns Angabe „Aria Königinn (319)“ auf Seite 319 der Arnold-Ausgabe: Dort beginnt die Arie „Will the sun forget to streak“ der Königin von Saba aus dem dritten Akt.

8 Brief vom 20.07.1829 an Carl Friedrich Zelter, in: Mendelssohn, Sämtliche Briefe, Bd. 1, hrsg. von Juliette Appold und Regina Back, Kassel u. a. 2008, S. 342.

$9 \mathrm{Zu}$ Mendelssohns Notizen vgl. Ralf Wehner, „Zu Mendelssohns Kenntnis Händelscher Werke“, in: Händel-Jahrbuch 53 (2007), S. 173-201, hier: S. 176 ff. und S. 190 ff. 
Der Standort der Partitur des Oratoriums, die Mendelssohn 1830 während seines Romaufenthalts „von Fortunato Santini erhielt und aufführungspraktisch einrichtete“, ${ }^{\circ}$ ließ sich bislang ebenso wenig ermitteln wie das Exemplar eines Klavierauszugs, in den Mendelssohn eine deutsche Übersetzung des Oratorientextes eintrug, die sein Freund Carl Klingemann erstellt hatte. ${ }^{11}$ Dies gilt auch für eine weitere Orgelstimme, die für den Kölner Appellationsgerichtsrat und Mendelssohn-Freund Erich Heinrich Verkenius, der das damalige Kölner Musikleben maßgeblich beeinflusste, ${ }^{12}$ angefertigt worden sein soll. ${ }^{13}$

In der Bibliothek der Hochschule für Musik und Tanz Köln konnte der Verfasser dieses Beitrags sowohl eine Orgelstimme zu Händels Salomon, mit zahlreichen Eintragungen von Mendelssohns Hand, als auch eine zweibändige, von Verkenius angefertigte Partiturabschrift der aufführungspraktischen Einrichtung Mendelssohns mit deutschem Text auffinden. ${ }^{14}$ Die Titelblätter beider Partiturbände enthalten folgenden Text, der ebenfalls von Verkenius geschrieben wurde: „Salomon, Oratorium von Händel, | mit Orgelbegleitung von Mendelssohn | so wie es 1835 beim Musikfest in Köln | aufgeführt wurde." Im von Verkenius erstellten Katalog seiner Musikbibliothek findet sich unter Nr. 291 ein Eintrag, der sich zweifellos auf die beiden genannten Partiturbände bezieht und auch den Verfasser der deutschen Übersetzung nennt: „Händel, G. Fr. Salomon, Oratorium für 4 Singstimmen und Orchester, mit deutschem Text von C Klingemann, und Orgelbegleitung von F. Mendelssohn. geschrieben." 15

\section{Orgelstimme}

Die wiederaufgefundene Orgelstimme, die in diesem Beitrag als Kölner Stimme bezeichnet wird, befindet sich in gutem Erhaltungszustand und ist mit Fäden in einen dunkelblauen Umschlag geheftet, der die beiden Stempelungen „Conservatorium der Musik Cöln“ und „Bücherei der staatl. Hochschule für Musik Köln“ trägt. Der handschriftliche Umschlagtitel enthält folgenden Text: „Salomon | Oratorium von Händel. | Orgelstimme“. Die Stimme wurde von einem unbekannten Kopisten angefertigt und besteht wie das Berliner Autograph aus nummerierten Sätzen, deren Zählung mit „52“ endet. ${ }^{16}$ Der Umfang der

10 Wehner, Felix Mendelssohn Bartholdy, S. 506. Ein Exemplar von Händels Solomon in der Ausgabe Arnolds, das sich im Besitz Santinis befand, wurde für diesen Beitrag verwendet, D-MÜs SANT Dr 367.

11 Vgl. Brief vom 21.01.1836 an Verkenius, in: Mendelssohn, Sämtliche Briefe, Bd. 4, S. 377.

12 Vgl. Imogen Fellinger, Art. „Verkenius, Erich Heinrich“, in: Rheinische Musiker 6, hrsg. von Dietrich Kämper (= Beiträge zur rheinischen Musikgeschichte 80), Köln 1969, S. 213 ff.

13 Vgl. Wehner, Felix Mendelssohn Bartholdy, S. 506.

14 D-KNh R 810 bzw. D-KNh R 2 und R 3. Aufgrund eines Schriftvergleichs mit diversen Briefen und anderen Partiturabschriften konnte Verkenius als Schreiber der Partitur zweifelsfrei identifiziert werden.

15 D-KNh L 348, Rubrik „Partituren“.

16 In den Fällen, in denen Mendelssohn keine eigenen Satzstreichungen vornahm, orientiert sich die Nummerierung in den Orgelstimmen am Klavierauszug, der 1831 in Bonn bei Simrock erschien, vgl. Salomon, großes Oratorium in drei Abtheilungen von G. F. Händel, mit frei übersetztem deutschem Texte, im Clavierauszuge von Xav. Gleichauf, Bonn 1831. So ist Zadoks Arie „See the tall Palm that lifts the head“ sowohl im Auszug als auch in beiden Stimmen unter „No. 32“ angeführt. Der Auszug lässt das folgende Rezitativ und die Arie des ersten Weibes, die Mendelssohn 1835 beide aufgeführt hat, aus und fährt direkt mit dem Chor "Swell the full chorus" fort, der mit „No. 33“ angegeben ist. Die Orgelstimmen notieren deshalb für das Rezitativ „No. 32 1⁄2“, während die Arie keine Nummerierung erhielt, der Chor aber wie im Klavierauszug als „No. 33“ gezählt wird. Die bereits genannte Arnold-Ausgabe enthält keine Satzzählung. 
Eintragungen Mendelssohns reicht von wenigen Korrekturen einzelner Noten über eine Vielzahl hinzugefügter Registrieranweisungen bis hin zur vollständigen Neubearbeitung einzelner Sätze. Vor allem in den Chören ergänzte er den Part für beide Hände. Zudem enthält die Kölner Stimme für die Arie „Thrice bless'd that wise discerning king“ und das Terzett „Words are weak to paint my fears" eine weitere Fassung des Orgelparts, die Mendelssohn in Reinschrift auf zwei eingelegten, losen Doppelbögen notierte, deren Papier mit dem Aufdruck „Bonn bei N. Simrock“ gekennzeichnet ist. Somit sind von diesen beiden Sätzen jeweils drei Fassungen überliefert: eine im Berliner Autograph und zwei in der Kölner Stimme.

$\mathrm{Zu}$ Mendelssohns Zeit war es in Deutschland üblich, Händels Oratorien ohne Orgelbegleitung und mit Hinzufügung von Blasinstrumenten aufzuführen. ${ }^{17}$ Entsprechende Einrichtungen Ignaz Franz von Mosels oder Johann Hermann Clasings waren insbesondere bei den Niederrheinischen Musikfesten 1830 und 1832 zu hören. ${ }^{18}$ Dagegen beabsichtigte Mendelssohn, Händels Salomon 1835 „in der ursprünglichen Gestalt mit Orgel“19 und „ohne weitre Bearbeitung und Instrumentirung von Herrn von Mosel oder irgend einem andern Dilettanten, der dafür später in der Hölle brät“, aufzuführen. „[...] die Orgel begleitet die Arien, als thäte es Händel selbst [...] und man wird zum erstenmale wieder den echten Händel hören. “20

Nachdem Mendelssohns Vorhaben beim Comité des Niederrheinischen Musikfests „allgemeinen Beifall“21 gefunden hatte, erkundigte er sich bei George Smart über die bei Händel-Aufführungen in England übliche Art der Orgelbegleitung. ${ }^{22}$ Den Entschluss, eine Orgelstimme für das Musikfest in Köln anzufertigen, fasste er auch aus aufführungspraktischen Gründen: „Die Orgel zum Salomon werde ich nicht selbst spielen können, da sie im Hintergrunde des Orchesters stehn muß, und fast alle Stücke begleitet, während die hiesigen Chöre und Spieler an ein fast immerwährendes Tactschlagen gewöhnt sind. Ich werde daher die ganze Orgelstimme in der Art wie ich sie mir gespielt denke, schreiben müssen, und der dortige Domorganist Weber wird sie spielen; er soll ein fester Musikus und guter Spieler sein, also geht das recht gut, macht mir nur die große Arbeit des Schreibens, da ich die Sache so gut wie möglich zu haben wünschte." 23 Wie aus einem Brief an seinen Vater hervorgeht, schloss Mendelssohn die Arbeit an der Orgelstimme am 23. Mai 1835 in Düsseldorf ab, ${ }^{24}$ doch musste er sie bereits wenige Tage später in weiten Teilen umarbeiten: „Mein Einfall mit der Orgel war der allerglücklichste, obwohl wir erst per aspera ad astra mußten, denn in der ersten Probe mit Chor, Orchester und Orgel klang und ging alles so entsetzlich schlecht, daß ich die ganze Idee und somit das ganze Fest für verrechnet ansah. Ein Paar Stellen hatten gut geklungen; auf die fußte ich noch, schrieb die halbe

17 Vgl. Mendelssohns Brief vom 24.03.1835 an George Smart, in: Mendelssohn, Sämtliche Briefe, Bd. 4, S. 198 sowie R. Larry Todd, Felix Mendelssohn Bartholdy: Sein Leben - seine Musik, Stuttgart 2008, S. 368 .

18 Vgl. Niemöller, S. 52.

19 Brief vom 10.04.1835 an Henriette Voigt, in: Mendelssohn, Sämtliche Briefe, Bd. 4, S. 222.

20 Brief vom 11.03.1835 an die Familie in Berlin, in: ebd., S. 187. Zur Diskussion um Mendelssohns „Werktreue“, vgl. z. B. Wolfgang Sandberger, „Händels Israel in Ägypten zwischen, Werktreue und kulturpolitischem Manifest: die Aufführungen unter Felix Mendelssohn Bartholdy in Düsseldorf 1833“, in: Göttinger Händel-Beiträge 13 (2010), S. 29-48, insbesondere S. 35.

21 Brief vom 10.03.1835 an die Familie, in: Mendelssohn, Sämtliche Briefe, Bd. 4, S. 187.

22 Vgl. Brief vom 24.03.1835 an George Smart, in: ebd., S. 198 f.

23 Brief vom 03.04.1835 an seine Eltern, in: ebd., S. 212.

24 Vgl. Brief vom 23.05.1835, in: ebd., S. 243. 
Orgelstimme in der Nacht danach um, brachte die Effecte hinein, die ich erst hatte kennen lernen, und bei der nächsten Probe bekam ich Oberwasser. " 25 Laut Plan sollte die erste gemeinsame „Probe des Salomon“ mit Chor und Orchester unter Mendelssohns Leitung am Montag, den 1. Juni stattfinden. ${ }^{26}$ Die Umarbeitung der Orgelstimme erfolgte somit wohl in der Nacht vom 1. auf den 2. Juni 1835, so dass Domorganist Franz Weber noch drei Tage Zeit blieben, um sie bis zur ersten Generalprobe am Freitag, den 5. Juni einzustudieren. Anhand der Kölner Stimme lässt sich dieser weitläufige Umarbeitungsprozess beobachten.

Da im Berliner Autograph vergleichsweise wenige Korrekturen vorhanden sind, gibt es die Orgelstimme vor ihrer Umarbeitung wieder. Es enthält Mendelssohns ausgeschriebenen Orgelpart, den der Kopist in die Kölner Stimme übertrug. Sollte die Orgelstimme allerdings der Partiturvorlage folgen, dann hat Mendelssohn den entsprechenden Abschnitt nicht vollständig abgeschrieben, sondern ihn mit Anweisungen für den Schreiber der Kölner Stimme versehen. So sind beispielsweise die Takte 8 bis 80 des Chores „Your harps and cymbals sound" nicht im Berliner Autograph enthalten, sondern durch folgenden Hinweis ersetzt: „vide Partitur von pag. 13 Tact 2, bis pag. 36 Tact 1.“ Der Kopist folgte dieser Anweisung und schrieb den bezifferten Bass aus der Vorlage ab. Seine Arbeit muss vor der ersten gemeinsamen Probe von Chor und Orchester am 1. Juni beendet gewesen sein. Weiterhin müssen sowohl Mendelssohn als auch der Kopist mit derselben Vorlage gearbeitet haben. Ein Vergleich ergab, dass sich die im Berliner Autograph angegebenen Seitenzahlen samt zugehörigen Taktangaben auf die bereits genannte Arnold-Partiturausgabe beziehen und diese den Orgelpart aufweist, den der Kopist nach Anweisung Mendelssohns abschrieb. ${ }^{27}$ Für die Aufführung 1835 konnte somit nur die Kölner Stimme Verwendung finden, da sie im Gegensatz zum Berliner Autograph den Orgelpart für alle vorgesehenen Sätze vollständig enthält.

Die Orgel, die Mendelssohn 1835 für seine Aufführung im Gürzenichsaal zur Verfügung gestellt wurde, ${ }^{28}$ stammte aus St. Kunibert (Köln), ${ }^{29}$ hatte mit zwei Manualen und Pedal einen Umfang von $C$ - $d$ ”' und war wie folgt disponiert: „1.) Bourdon 16 Fuß 2.) Octav 8 Fuß. 3.) Hohlpfeiff 8 Fuß. 4.) Octav 4 Fuß 5.) Gamba 8 Fuß. 6.) Supperoctav 2 Fuß. 7.) Mixtur 4 Fach. 8.) Trompett 8 Fuß. 9.) Claron Bass 4 Fuß.“ 30

25 Brief vom 26.06.1835 an Klingemann, in: ebd., S. $254 \mathrm{f}$.

26 Vgl. D-KNmi, Archiv für Rheinische Musikgeschichte, A/I/21/1.42, Protokoll des Musikfestcomités vom 21.05.1835. Nach dieser ersten gemeinsamen Probe sollten an zwei weiteren Tagen (02.06. bzw. 04.06.) andere für das Musikfest vorgesehene Werke einstudiert werden. Es folgte die „erste GeneralProbe“ am Freitag, den 05.06., bei der bereits Publikum zugelassen war, vgl. Niemöller, S. 62. Die letzten "General-Proben“ vor der Aufführung des Salomon (07.06.) fanden am Samstag, den 6. Juni „Morgens und Nachmittags" statt. Mendelssohn hatte somit an drei Tagen Gelegenheit, das Oratorium mit Chor und Orchester gemeinsam einzustudieren: am 1., 5. und 6. Juni 1835.

27 Im Gegensatz zur Arnold-Ausgabe ist der Part in beiden Orgelstimmen auf zwei Systeme verteilt und im Violin- und Bassschlüssel verzeichnet. Mendelssohn korrigierte aus der Arnold-Ausgabe falsch abgeschriebene Noten und ergänzte gelegentlich einzelne Töne und Akkorde, um den Übergang zu Tastosolo-Passagen abzurunden.

28 Beim Musikfest 1838 wurde die Orgel unter Mendelssohns Leitung erneut verwendet und um ein Posaune-16'-Register erweitert, vgl. Joseph Esser, Felix Mendelssohn Bartholdy und die Rheinlande, Diss. Bonn [1923], S. 56.

$29 \mathrm{Vgl}$. Niemöller, S. $53 \mathrm{f}$.

30 Angaben nach dem von Orgelbauer Engelbert Maaß am 02.06 .1835 erstellten „Verzeichniß ueber die erhaltenen Gegenstände von der Orgel zu St Cunibert in Cöln, welche zum Gebrauch einer Orgel für das Niederrheinische Musikfest auf den Saal Gürzenich gestellt worden sind“, D-KNmi, Archiv für Rheinische Musikgeschichte, A/I/18/1.16. Mendelssohns Angabe „es kommen 2mal 16 Fuß hinein“ 
Nach der missglückten ersten gemeinsamen Probe mit dem groß besetzten Ensemble des Musikfests ${ }^{31}$ begann Mendelssohn mit verschiedenen Registerkombinationen zu experimentieren. Diese Arbeit führte ihn schließlich zu einem Schlüsselerlebnis besonderer Art: „Das Ding fing an zu klingen, mit ein Paar Zusätzen von 16 Fuß hier, 4 Fuß dort bekam es auf einmal solche Wirkung, daß in der dritten Probe alles entzückt war, und ich muß

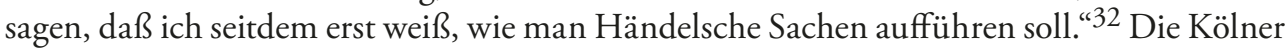
Orgelstimme dokumentiert diese Experimente, denn sie verzeichnet die Registrierungsangaben, die Mendelssohn erwähnt: „Namentlich der Chor ,draw the tear 'klang mit Prinzipal 8 F., und Bourdun 16 F. in dicken Akkordlagen wunderschön“33 (vgl. Abbildung 1).

Händel gestaltet den Beginn dieses Chores („Largo“) als fünfstimmige Fuge, die von gehaltenen Streicherakkorden begleitet wird (T. 1-14, Themeneinsätze der Vokalstimmen: T, A, S II, B, S I, B). Während im Berliner Autograph zu diesem Chor lediglich eine Anweisung für den Kopisten vorhanden ist („No. 41 Chor vide Part. p. 265.“), weist die Kölner Stimme neben dem aus der Arnold-Ausgabe kopierten bezifferten Bass auch einen akkordischen Part für die rechte und linke Hand auf, den Mendelssohn in der Nacht nach der ersten gemeinsamen Probe in Tinte ergänzte. Die mit Bleistift geschriebene Registrieranweisung „16 Fuss und Gambe“ in T. 1 ff. wurde wohl erst während der folgenden Generalproben vom Organisten Franz Weber auf Anweisung Mendelssohns nachgetragen. ${ }^{34}$ Ursprünglich sollte an dieser Stelle die Registrierung des vorangehenden Rezitativs „Viola da Gamba pp" unverändert beibehalten werden, bis mit den beiden Themeneinsätzen des Vokalbasses „Principal“ (T. 8) und „16 Fuß“ (T. 12) hinzutreten. ${ }^{35}$ In den Generalproben dürfte sich herausgestellt haben, dass sich die anfängliche Registrierung nur mit „Viola da Gamba" kaum von den gehaltenen Streicherakkorden abheben und gegen die wachsende Zahl der Vokalstimmen durchsetzen konnte. Wohl aus diesem Grund wies er Franz Weber

wurde demnach nicht realisiert, vgl. Brief vom 23.03.1835 an seinen Vater, in: Mendelssohn, Sämtliche Briefe, Bd. 4, S. 197. Laut Niemöller, S. 54, wurden zwei der ursprünglich vorhandenen Register, Trompett 8' und Claron Baß 4', „nicht hinzugenommen“. Maaß hatte mündlich erklärt, dass „,eine Ersparniß von circa 5 Thlr eintreten “könne, „wenn [...] Trompett und Claron fortblieben“, vgl. D-KNmi, Archiv für Rheinische Musikgeschichte, A/I/18/1.12, Eintrag vom 24.04.1835. Tatsächlich sparte man Kosten bezüglich der Orgel, doch geht aus den Dokumenten nicht hervor, ob dies beide Register betraf, vgl. Eintrag vom 03.05. in A/I/18/1.15 vom 23.04. mit „Quittung“, A/II/22/1.176 vom 17.06.1835 und „Büdget 1835“, A/I/18/1.9. Zudem gibt Maaß in seinem genannten „Verzeichniß“ an, dass beide Register in den Gürzenichsaal transportiert wurden. Das „Verzeichniß“ wurde am 2. Juni, einen Tag nach der ersten gemeinsamen Probe von Chor und Orchester bzw. nur wenige Tage vor der Aufführung, erstellt. Weiterhin findet sich in der Kölner Stimme in der Ouverture, die „mit Mixtur volles Werk“ beginnt, beim ersten Basseinsatz im Allegro-moderato-Abschnitt, eine mit Bleistift eingetragene Registrieranweisung „ohne Zungen“, die Verkenius in seine Partiturabschrift übernahm: „ohne Zungenwerke“. Der Eintrag kann daher nicht in wesentlich späterer Zeit oder gar für eine andere Aufführung vorgenommen worden sein. Die Entstehungszeit der Partitur wird weiter unten im Abschnitt „Partitur“ erläutert. Verkenius war auch an der Planung der Orgel beteiligt, vgl. A/I/18/13.

31 Das Ensemble bestand aus 427 Chorsängern und 179 Instrumentalisten, vgl. Niemöller, S. 60.

32 Brief vom 26.06.1835 an Klingemann, in: Mendelssohn, Sämtliche Briefe, Bd. 4, S. 255.

33 Ebd.

34 Diese Registrieranweisung kann nicht in wesentlich späterer Zeit nachgetragen worden sein, denn Verkenius hat sie in seine Partiturabschrift übernommen, schrieb aber „Prinzipal 16 F. u. Gambe.“ Da die Orgel kein weiteres 16'-Register, sondern nur Bourdun 16' enthielt und die Angabe 16' deshalb in der gesamten Orgelstimme nicht weiter differenziert wird, dürfte es sich um ein Missverständnis handeln. Zudem erwähnt Mendelssohn die Verwendung des Bourdun 16' in seinem Brief an Klingemann, s. o.

35 Angaben wie „Principal“ oder „Praestant“ meinen das Oktav-8’-Register der Orgel. 


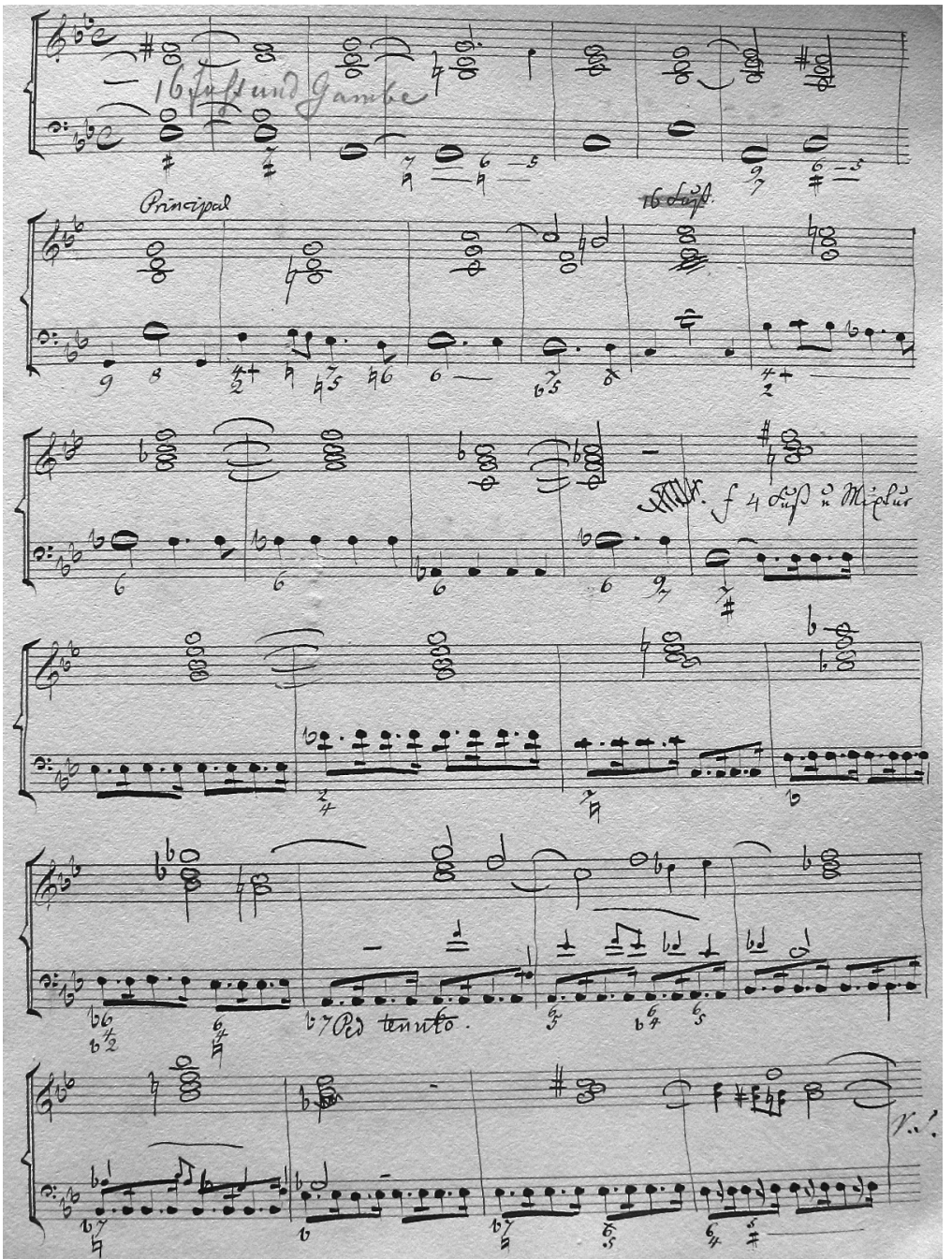

Abbildung 1: D-KNh R 810, Chor „Draw the tear“, T. 1-30

in den Proben an, das 16'-Register nicht erst in T. 12, sondern bereits in T. 1 zu ziehen, um eine Grundierung der Fuge ,in dicken Akkordlagen“ von Anfang an zu ermöglichen. Weber strich mit Bleistift Mendelssohns Anweisung in T. 12 und notierte in T. 1 „16 Fuss“ sowie „und Gambe“, um zu verdeutlichen, dass dieses Register nach wie vor aus dem vorherigen Rezitativ beibehalten werden sollte.

Nach Ende der Fuge (T. 14) werden die sich anschließenden Akkordblöcke der Vokalstimmen durch vollere Akkorde der rechten Hand begleitet (T. 14 ff.), bevor beim überraschenden D-Dur-Septakkord auf „death“ (T. 18) nach vorangehender Pause (T. 17) Mixtur und 4 ' im Forte umso wirkungsvoller einsetzen. ${ }^{36}$ Das Crescendo, das durch Aufregistrie-

36 Mendelssohn strich an dieser Stelle auch den ursprünglichen Tasto-solo-Vermerk („T. S.“). Die Pause konnte zum Ziehen beider Register genutzt werden. 
ren und Hinzutreten vollerer Akkorde entsteht, reicht bis zu einem neuen, vom punktierten Rhythmus des Orchesters geprägten Formteil, der ab T. 18 beginnt. In diesem neuem Abschnitt behält Mendelssohn sowohl die Registrierung mit „4 Fuß u Mixtur“ als auch die gehaltenen Akkorde bei, so dass die Orgel während der Pausen des Chores immer wieder hervortritt. Ohne vorangehende Zäsur lässt Händel das Fugenthema erneut in die unteren Vokalstimmen einfließen (Tenor, T. 24 ff. und Bass, T. 26 ff.), während die oberen zugleich nichtthematische Einsätze beisteuern und die Streicher unablässig in punktierten Rhythmen begleiten. Im Gegensatz zum klar erkennbaren Nacheinander der Themeneinsätze in der Exposition (T. 1 ff.), bestand an dieser Stelle die Gefahr, dass das Fugenthema durch den massiven Klang der übrigen Vokalstimmen und des Orchesters völlig „zugedeckt“ wird. Wohl um dies zu vermeiden, zeichnet Mendelssohns Orgelpart beide Themeneinsätze in der linken Hand nach (T. 24 ff. und 26 ff.). ${ }^{37}$ Die Art dieser Modifikation erforderte auch den Einsatz des Pedals, um eine Spielbarkeit des Basses zu gewährleisten (T. 24, „Ped tenuto").

Gegen Ende des Satzes lässt Händel das Fugenthema nochmals erscheinen, gibt allerdings gleichzeitig den punktierten Rhythmus zugunsten gehaltener Streicherakkorde auf. Da dieses Zusammentreffen von Fugenthema und Streicherakkorden sehr an die Gestaltung der Exposition zu Beginn des Satzes erinnert (T. 1-14), kehrt der Orgelpart nach vorangehender Pause (T. 31) wieder zur vormaligen Registrierung zurück („ohne 4 Fuß u Mixtur"), so dass eine Decrescendo-Wirkung entsteht. Wenige Takte darauf lässt Mendelssohn die Orgel während der gesamten Generalpause (T. 36 f.) klingen. Eine solche Überbrückung von Pausen zählte, neben dem treffsicheren Einsatz der Mixtur (T. 18), vermutlich zu den klanglichen „Effecten“, die er während der Probenarbeit „erst hatte kennen lernen" müssen.

Da sich in der Arnold-Partitur für diesen Chor keinerlei Angaben zur Dynamik finden, sah sich Mendelssohn mit einer Problematik konfrontiert, mit der man sich zu seiner Zeit bei Aufführungsvorbereitungen von Werken des Barock auseinanderzusetzen hatte: Waren keine Vortragsbezeichnungen vorhanden, dann wurde es notwendig, entsprechende Einzeichnungen vorzunehmen. ${ }^{38}$ Wie Mendelssohn sich die dynamische Gestaltung dieses Händelsatzes grundsätzlich dachte, zeigt die durch Auf- und Abregistrieren erzielte Crescendo- und Decrescendo-Wirkung, die auf eine seinerzeit favorisierte, fließende Dynamik hindeutet: „Die Einsätze waren fest, die Forte's kräftig, die Piano's zart, die Crescendo's und Diminuendo's ebenmäßig (nicht springend, wie man es so oft hört), kurz, Alles so, wie es sich gebührt. " ${ }^{39}$ Vermutlich steht diese Dynamik auch mit einer bei den damaligen Niederrheinischen Musikfesten favorisierten Aufführungspraxis in Zusammenhang: „Das Instrumental-Orchester war in einem spitzen Winkel zwischen dem Sänger-Personal bis an das Directionspult vorgeschoben, und diese Spitze enthielt vorzugsweise die zur SoloBegleitung und für die Piano's bestimmten Instrumente. Auch die Blas-Instrumente für die

37 Ein vergleichbares „Herausarbeiten“ des Fugenthemas lässt sich auch in Mendelssohns Bearbeitung des Eingangschors von Bachs Kantate BWV 43 beobachten, vgl. Anselm Hartinger, „Felix Mendelssohn Bartholdy und Bachs ,Himmelfahrtskantate' auf dem Kölner Musikfest 1838 - aufführungspraktische, quellenkundliche und ästhetische Konnotationen“, in: „Zu groß, zu unerreichbar“", Bach-Rezeption im Zeitalter Mendelssohns und Schumanns, hrsg. von Anselm Hartinger, Christoph Wolff und Peter Wollny, Wiesbaden 2007, S. 281-314, hier: S. $300 \mathrm{ff}$.

38 Vgl. ebd., S. 283.

39 A. J. Becher, „Das niederrheinische Musikfest für 1835“, in: Beiblatt der Kölnischen Zeitung 12 (1835), ohne Seitenangabe. 
Soli schlossen sich an dieses kleinere Orchester an, und neben und hinter ihnen breiteten sich die Massen der übrigen Ripien-Instrumente aus. Alle Pianostellen, selbst in den Symphonieen, wurden ausschliesslich von dem kleineren Solo-Orchester vorgetragen, welches dann im Crescendo und Forte durch die Ripien-Pulte mehr und mehr bis zum Fortissimo der ganzen Masse verstärkt wurde. “40 Falls Mendelssohn 1835 dieser Praxis auch für Salomon gefolgt sein sollte, ${ }^{41}$ dann dürfte die Registrierung des Orgelparts von einer solchen Concertino-Ripieno-Dynamik beeinflusst sein.

In dem bereits erwähnten Brief an Smart erkundigt sich Mendelssohn nach der Behandlung der Orgel am Schluss der Chöre: „I know that the full organ comes in always towards the end of the Choruses which produces a very good effect, but I should like to know whether such places are marked or entirely left to the choice of the organist, and whether there are any other rules followed by the organ player in the Oratorios. ${ }^{\text {“42 }}$ In der Kölner Stimme ist dieser „Effect“ besonders ausgeprägt: Während in der Berliner Stimme für den Chor „From the censer curling rise“ lediglich die Anweisung für den Kopisten „No. 19 siehe Partitur" vorhanden ist, ergänzte Mendelssohn ab T. 100 bis Schluss volle Akkorde im Fortissimo für die rechte und linke Hand:

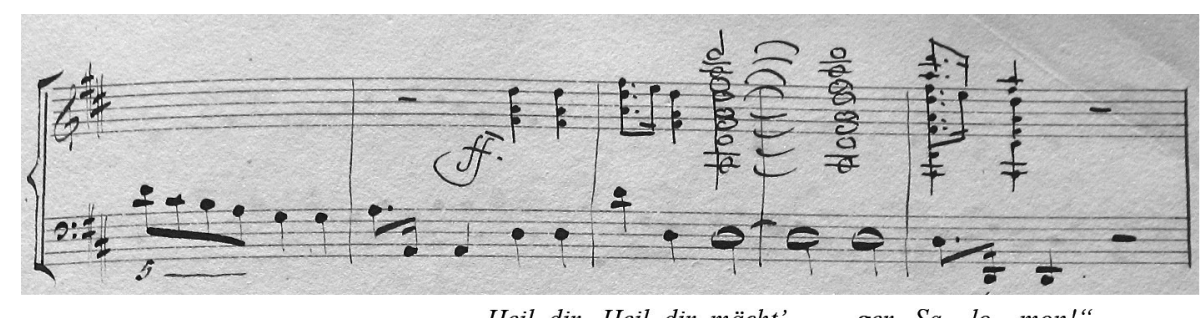

"Heil dir, Heil dir, mächt’_ger Sa-lo-mon!"

Abbildung 2: D-KNh R 810, Chor „From the censer“, T. 99-103

In Händels Chor setzen die Jubelrufe „Live for ever, mighty Solomon!“ („Heil dir, Heil dir, mächt'ger Salomon!“) teilweise recht überraschend ein, indem sie den Fluss rein orchestraler Passagen abrupt unterbrechen (T. 108 ff. und 115 ff.). Die Orgel begleitet mit vollen Akkorden zunächst nur diese Rufe (vgl. Abbildung 2), wohl um den „Überraschungseffekt" mit großer Wirkung herauszustellen und einen schlagkräftigen Einsatz möglichst aller Chorsänger zu befördern. Schlagkraft, Wucht und „Knallen“43 der Masse gehörten auch zu ästhetischen Erwartungen, die man bei den Niederrheinischen Musikfesten jener Zeit an ein Chorwerk stellte. ${ }^{44}$

Für den Chor „Throughout the land Jehovah's praise“ findet sich im Berliner Autograph ebenfalls nur die Angabe „No 8 Coro vide Partitur“. In der Kölner Stimme schweigt

40 [Wilhelm Hauchecorne], Blätter der Erinnerung an die fünfzigjährige Dauer der Niederrheinischen Musikfeste, Köln 1868, S. 28.

41 Vgl. Niemöller, S. 62 f. Hartinger (S. 305) lässt dies für das von Mendelssohn geleitete Musikfest 1838 offen.

42 Brief vom 24.03.1835, in: Mendelssohn, Sämtliche Briefe, Bd. 4, S. 198. Smarts Antwortschreiben hat sich nach bisherigem Forschungsstand nicht erhalten, vgl. Susanna Großmann-Vendrey, Felix Mendelssohn Bartholdy und die Musik der Vergangenheit, Regensburg 1969, S. 78 sowie Wm. A. Little, Mendelssohn and the Organ, Oxford 2010, S. 153.

43 So Mendelssohn in einem Brief vom 09.12.1837 an Franz Hauser, zit. nach Hartinger, S. 303.

44 Ebd. S. 303 f. 
die Orgel ab T. 77 („senza Organo“), um mit Beginn der letzten Engführungsphase ab T. $101 \mathrm{ff}$. (Bass, Sopran II und I) umso wirkungsvoller einzusetzen. Gegen Ende des Satzes steigert Mendelssohn die Schlusswirkung, indem er die Begleitung im „Fortissimo“ zu siebentönigen Akkorden auffächert. Derartige „Effekte“ der Orgel erregten bei der Aufführung 1835 besondere Aufmerksamkeit: „Die Wirkung war außerordentlich, sowohl in den Chören, wenn das volle Werk, besonders in einigen Nummern gegen den Schluß, wo der Componist alle seine Kräfte zu verdoppeln strebt, gewaltig erbraus'te“. 45

Als Ferdinand Hiller im Rahmen des 39. Niederrheinischen Musikfests am 8. Juni 1862 Händels Salomon unter Verwendung von Mendelssohns Stimme und Verkenius' Partiturabschrift erneut in Köln aufführte, ${ }^{46}$ wurde die Wirkung der gehaltenen Akkorde und die besondere Behandlung der Pausen bemerkt: „Zu allen vier Chören [ab Beginn des dritten Teils] hat Mendelssohn die Orgel fast immer obligat und mehrstimmig gesetzt und dadurch namentlich in den ersten drei hinreissende Wirkungen erzeugt, [...] im Kriegerchor [„Shake the dome“] durch den Gedanken, das volle Werk in gehaltenen Accorden mit Zwischenpausen von ganzen oder halben Tacten darein klingen zu lassen. “47

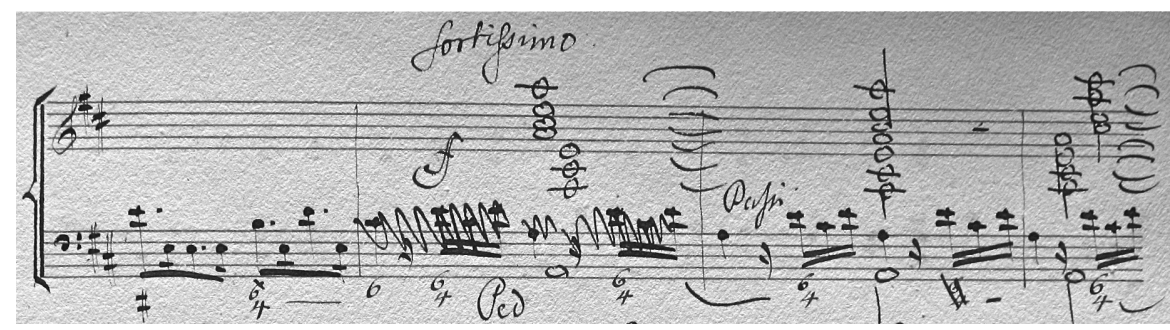

Abbildung 3: D-KNh R 810, Chor „Shake the dome“, T. 11-14

Nachdem die Orgel bis T. 11 des Chores lediglich mezzoforte und tasto solo begleitet, setzen gehaltene Akkorde mit Pedal im vollen Werk ab T. 12 ein („fortissimo“). Bei Händel beginnt an dieser Stelle ein Schlagabtausch beider Vokalchöre, der von Motiveinwürfen der Oboen und Streicher in Sechzehnteln begleitet wird, die über weite Strecken akkordeigen figurieren. In der Kölner Stimme strich Mendelssohn die Figuration der Bassstimme zugunsten massiver Akkorde und halbtaktiger Pausen (vgl. Abbildung 3), so dass der Orgel eine von der Führung des Generalbasses unabhängige Funktion zugewiesen wird. ${ }^{48} \mathrm{Zu}$ dem lassen die gehaltenen Akkorde die seinerzeit übliche Hinzufügung zusätzlicher Blasinstrumente überflüssig erscheinen.

Der Wechsel von Akkorden und Pausen (T. 12-19) wird auch an den Parallelstellen erneut aufgegriffen (T. 25-28 und 31-38) und somit großflächig der lebhaften Figuration

45 Becher, ohne Seitenangabe.

46 Vgl. Art. „Das 39. niederrheinische Musikfest“, in: Niederrheinische Musik-Zeitung für Kunstfreunde und Künstler 10 (1862), S. 185, linke Spalte und S. 188 f.

47 Ebd., S. 193, rechte Spalte, Einfügungen vom Verfasser dieses Beitrags. Indes gilt es zu berücksichtigen, dass zu diesem Anlass eine „von Ibach Söhne in Barmen“ eigens erbaute Orgel mit 21 Registern verwendet wurde und die klanglichen Verhältnisse deshalb andere waren, vgl. ebd., S. 188, linke Spalte. Die Orgel wurde 1862 erneut von Franz Weber gespielt, vgl. ebd.

48 Vgl. die ähnliche Feststellung bei Ralf Wehner, „Mendelssohn and the Performance of Handel's Vocal Works", in: Mendelssohn in Performance, hrsg. von Siegwart Reichwald, Bloomington 2009, S. 147170, hier: S. 154: „Mendelssohn based his organ parts somewhat on Handel's figured bass, but deviated when he felt it necessary." 
des Satzes entgegengestellt. Diese Tendenz hin zur Glättung und Vereinheitlichung des Klanggeschehens könnte ebenfalls mit bestimmten seinerzeit favorisierten Klangidealen in Verbindung stehen: In einem Bericht über das Musikfest 1837, das unter Leitung von Ferdinand Ries stand, wird die „größere Wirkung“ von „Glätte und Gleichheit“ herausgestellt, wenn sie „in die gesamte Masse“ hineingearbeitet würde. ${ }^{49}$

Im Chor „Praise the Lord“ aus dem dritten Teil zitiert „Händel bei den Worten ,God alone is just and wise' eine Melodie aus Luthers ,Deutschem Sanctus' [...] ,Heilig ist Gott, der Herre Zebaoth“"50 (ab T. 79). Im Berliner Autograph beginnt Mendelssohns eigene Orgelstimme erst an dieser Stelle, nachdem für alle vorigen Takte zunächst der Part aus der Arnold-Ausgabe vorgesehen war:
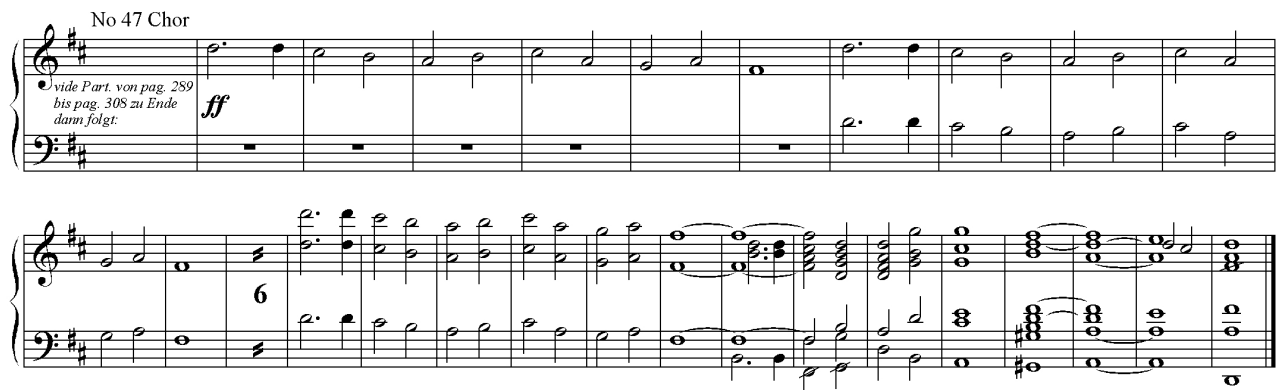

Abbildung 4a: D-B Mus. ms. autogr. Mendelssohn Bartholdy F. 28, S. 275, Chor „Praise the Lord“, T. 79-110

Dagegen vermerkt die Kölner Stimme für die Orchestereinleitung „senza Org.“, bevor beim ersten Einsatz der Vokalstimmen ab T. 7 die Orgel im Forte mit 4' ohne Mixtur hinzutritt. ${ }^{51}$ Im weiteren Verlauf erscheinen von Mendelssohn ergänzte akkordische Passagen im Wechsel mit „senza organo“-Anweisungen, bis schließlich der Cantus firmus ab T. 79 im vollen Werk einsetzt („,fortissimo“) (vgl. Abbildung 4b):

Im Gegensatz zur Berliner Stimme wird er von Anfang an hochoktaviert („8va alta“), im Oktavabstand verdoppelt und durch eine akkordische Schicht ergänzt (vgl. Abbildung 4a mit Abbildung 4b). ${ }^{52}$ Zugleich wird sein Beginn durch den höchsten Ton der Mendelssohn zur Verfügung stehenden Orgel markiert ( $d$ '”). Ab dem zweiten Cantus-firmus-Einsatz (T. 85) treten vermehrt akkordeigene Töne hinzu, derweil sich die linke Hand in tieferer Lage bewegt, bis der Part des Pedals am Ende des Chores schließlich zum tiefen $D$ hinabsinkt. Somit wird der mehrmalige Einsatz des Cantus firmus klanglich aufgefächert und in einen großen Bogen gefasst, dessen Ambitus sich vom d"' zu Beginn bis zum $D$ am Schluss erweitert.

Auch diese Modifikationen dürften auf Mendelssohns erste Probenerfahrungen mit dem Musikfestensemble zurückzuführen sein: In Händels zweichörig angelegtem Vokalsatz erscheint der Cantus firmus als Unisono des einen Chores, während der andere im vierstimmigen Satz kontrapunktiert. Im Gegensatz zum Berliner Orgelpart, der überwie-

49 Art. vom 18.06.1837, in: Der Verkündiger am Rhein oder Blätter für Politik, Literatur, Kunst und Weltleben, 23 (1837), zit. nach Hartinger, S. 305.

50 Hans Joachim Marx, Händels Oratorien, Oden und Serenaten, Göttingen 1998, S. 222.

51 Aus Verkenius' Partiturabschrift geht hervor, dass hiermit eine Registrierung 16', 8', 4' gemeint ist.

52 In der Ouvertüre wendet Mendelssohn ein ähnliches Verfahren an: Bereits der erste Akkord des ersten Taktes wird durch hochoktavierte, akkordeigene Töne ergänzt. 


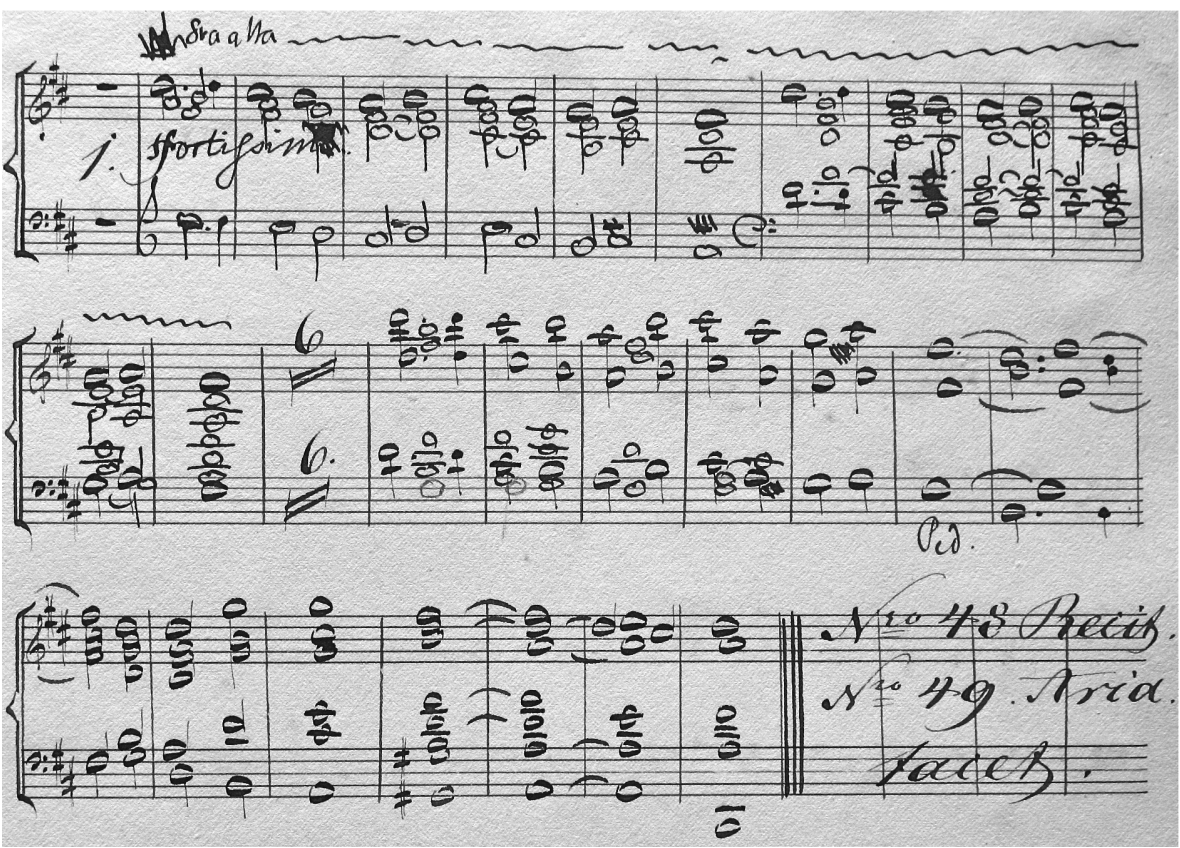

Abbildung 4b: D-KNh R 810, Chor „Praise the Lord“, T. 78-110

gend das Chorunisono verstärkt, hebt sich die akkordisch-homophone Begleitung der Kölner Stimme sowohl vom Unisono des einen als auch vom Kontrapunktieren des anderen Chores ab. Mittels Hochoktavierung, Oktavverdopplung und voller Akkorde konnte sich der Orgelpart wohl erst gegen den massiven Klang des Ensembles durchsetzen; die Berliner Stimme wirkt dagegen vergleichsweise „dünn“.

Für die Chöre „Music, spread thy voice around“ und „May no rash intruder“ fügte Mendelssohn den Part für beide Hände hinzu.

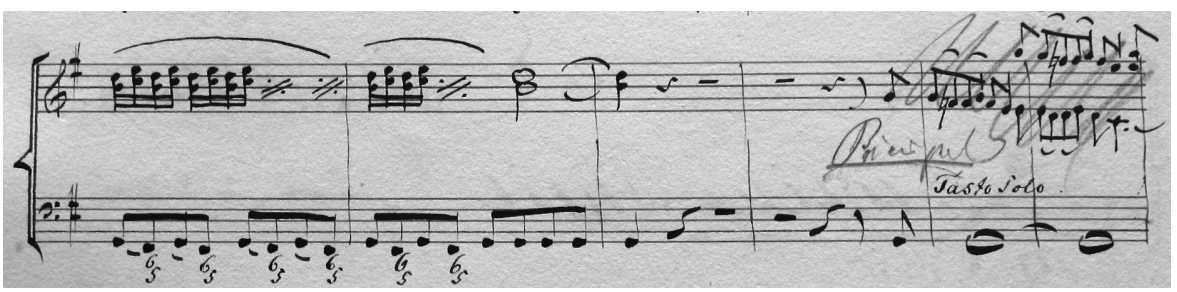

Abbildung 5: D-KNh R 810, Chor „May no rash intruder“, T. 40-45

In „May no rash intruder“ tragen in T. $40 \mathrm{f}$. Alt, Tenor und Bass den Text „ye zephirs, soft-breathing, their slumbers prolong“" ohne Beteiligung von Sopran I und II vor. Der tiefliegende Vokalpart wird von Sechzehntelfigurationen der Streicher umspielt, um das sanfte Säuseln des Zephyrs nachzuzeichnen. Mendelssohns Stimme (ab T. 39: „pp“) oktaviert diese Figuration in die Höhe, so dass der Orgelpart über Händels Satz wie ein zarter Silberstreif schimmert (vgl. Abbildung 5, T. 40 f.). Zunächst sollte die Orgel auch die kanonischen Einsätze der Singstimmen begleiten (T. 33 ff., 44 ff. und 50 ff.), doch wurde dies 
mit Bleistift wieder gestrichen, ${ }^{53}$ wohl um die Aufmerksamkeit auf bestimmte Haltetöne zu lenken (vgl. Abbildung 5, T. 44 f.), die durch Registrierung mit „Principal“ hervorgehoben wurden und einige Takte des Satzes umspannen.

Im Schlusschor „The name of the wicked shall quickly be past“ ergänzte Mendelssohn den Part für die rechte Hand und orientierte sich dabei vermutlich auch am Text: Während bei „Der Name des Bösen muß schnelle vergehn“ lediglich der vom Kopisten abgeschriebene bezifferte Bass gegeben ist, fügte Mendelssohn Akkorde im Forte mit „4 F.[uß] und Mixtur" bei „doch der Ruhm des Gerechten wird ewig bestehn“ hinzu. Erst nachdem sich beide Vokalchöre miteinander vereinen, ist für die letzten 20 Takte „fortissimo“ vorgeschrieben, bis gegen Ende des Satzes volle Akkorde mit Pedal einsetzen.

Auch innerhalb einiger Rezitative des zweiten und dritten Teils tritt die Orgel besonders hervor: ${ }^{54}$ So wird das erste Rezitativ der Königin von Saba signalartig mit einem einzelnen B-Dur-Akkord eröffnet („16’, 8', Hohlfl[öte] und Gambe“). Von Salomos Solo „Music, spread thy voice around“ bis einschließlich zum Chor „Thus rolling surges rise“ begleitet die Orgel in allen Sätzen ${ }^{55}$ und übernimmt somit für diese tableauartige Chorszene einheitsstiftende Funktion.

Im zweiten Teil werden neben Salomos strengem Spruch „Hear me, ye women, and the king regard“ auch alle Rezitativpartien des ersten Weibes bis zu „Israel, attend to what your king shall say“ von der Orgel in gehaltenen Akkorden begleitet. Für dieses Rezitativ sah Mendelssohn wohl deshalb keinen eigenen Orgelpart vor, weil Salomos Urteil bereits durch Händels Streicherbegleitung akzentuiert wird. Die Orgelstimme hebt somit Worte Salomos und des ersten Weibes hervor, um sie der Partie des zweiten Weibes kontrastierend gegenüberzustellen. ${ }^{56}$

Ähnliches lässt sich auch im Terzett „Words are weak“ beobachten, das, wie bereits erwähnt, in der Kölner Stimme in zwei Fassungen überliefert ist, die von Anbeginn unterschiedliche Konzepte aufweisen:

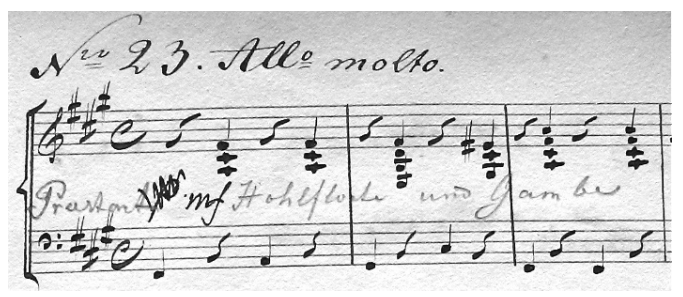

Abbildung 6a: D-KNh R 810, Zwischenfassung des Terzetts „Words are weak“, T. 1-3

53 Diese im weiteren Verlauf nicht leicht zu deutenden Streichungen wurden von Verkenius in seiner Partiturabschrift überwiegend berücksichtigt.

54 Dies ist auch im Berliner Autograph der Fall. Wolffs Angabe „this organ part is meant only for the arias and choral numbers, while the recitatives, here as well as in Israel in Egypt, are not accompanied by the organ“ ist nicht aufrechtzuerhalten, vgl. Hellmuth Christian Wolff, „Mendelssohn and Handel“, in: MQ 45 (1959), S. 175-190, hier: S. 184.

55 Dies gilt somit auch für die beiden Secco-Rezitative „Then at once from rage remove“ bzw. „Next the tortur'd soul release".

56 Es ist daher kaum verwunderlich, dass Mendelssohn für die Arie des zweiten Weibes „Thy sentence, great king" keine Orgelbegleitung vorsah. 


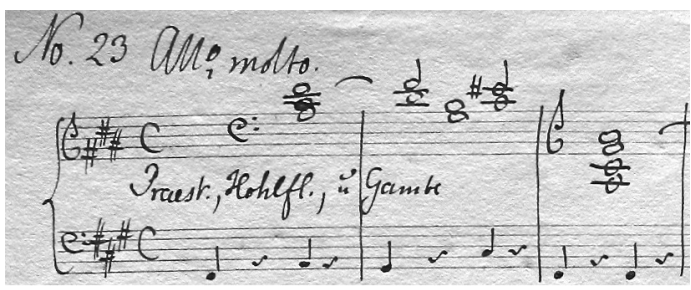

Abbildung 6b: D-KNh R 810, Reinschrift des Terzetts „Words are weak“, T. 1-3

Während Mendelssohn die in Bleistift ergänzte Registrierung „Praestant Hohlfloete und Gambe“ in seine Reinschrift übernahm, ersetzte er die nachschlagenden Viertel der rechten Hand durch gehaltene Akkorde, die nicht nur eine Grundierung für das pausendurchsetzte Orchesterritornell bilden (T. 1-13), sondern auch den Gesang des ersten Weibes hervorheben (T. 13-40), bevor die Orgel mit Einsatz des zweiten (T. 41-46) überraschend schweigt. Im Vergleich zum ursprünglich pausenreichen Part der rechten Hand, ist das Verstummen der Orgel nach gehaltenen Akkorden ungleich wirkungsvoller. Bei Salomos Gesang „justice holds the lifted scale“ (T. 46 ff., 54 ff., 63 ff. und 80 ff.) finden sich in der Zwischenfassung der Kölner Stimme zahlreiche Streichungen und Änderungen einzelner Töne in der Bassund in den Mittelstimmen:

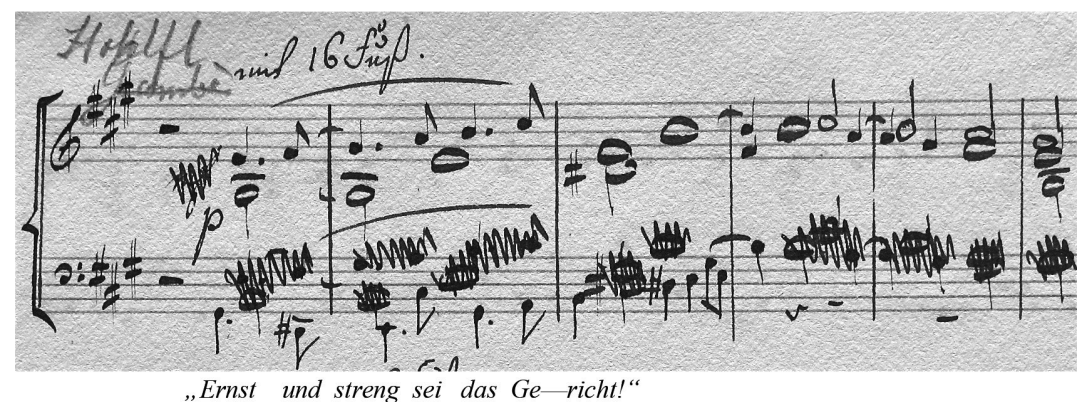

Abbildung 7: D-KNh R 810, Zwischenfassung des Terzetts „Words are weak“, T. 46-51

Im Vergleich zum Berliner Orgelpart, der den Klang zu sechstönigen Akkorden auffächert, erscheint die Kölner Stimme an diesen Stellen deutlich reduzierter. Dies geschah wohl auch deshalb, weil Mendelssohn sich entschloss, erstmals das 16'-Register in diesem Satz einzusetzen, das größere Klangfülle bot, um Salomos warnende Worte zu unterstreichen. ${ }^{57}$

In der Arie „Thrice bless'd that wise discerning king“ zeigen sich im Vergleich zur Berliner Stimme ebenfalls deutliche Unterschiede: ${ }^{58}$

57 Weber ergänzte noch „Hohlf[öte]“, um zu verdeutlichen, dass dieses Register nach wie vor beibehalten werden sollte. Die Registrierung wurde von Mendelssohn in die auf losem Bogen notierte Reinschrift übernommen.

58 Die zahlreichen Korrekturen in der Zwischenfassung der Kölner Stimme können in diesem Beitrag nicht weiter untersucht werden. Daher wird das Berliner Autograph nachfolgend nur mit der letzten, auf losem Bogen notierten Fassung der Kölner Stimme verglichen. 


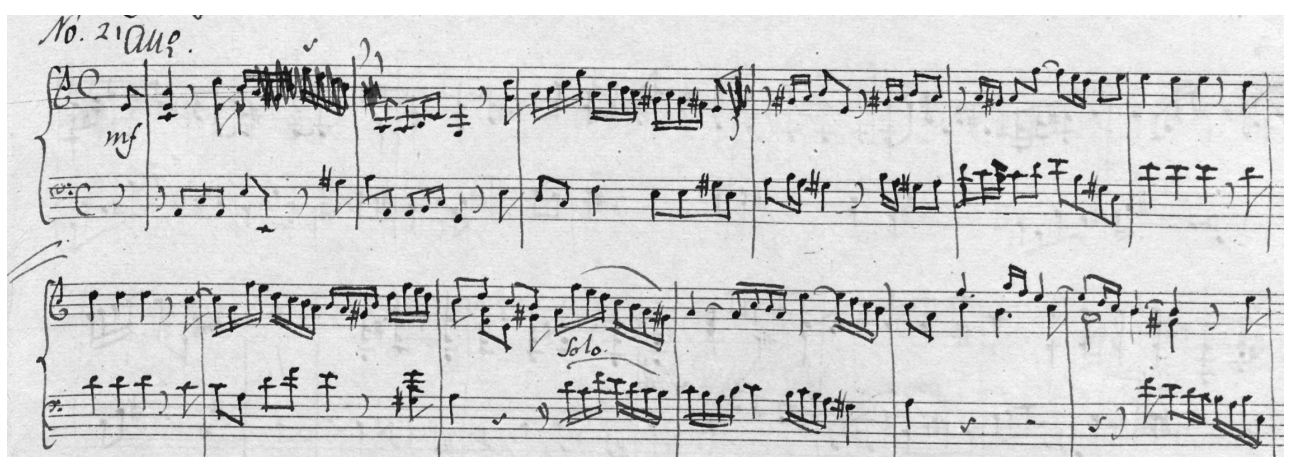

Abbildung 8a: D-B Mus. ms. autogr. Mendelssohn Bartholdy F. 28, S. 267, Arie „Thrice bless'd that wise“, T. 1-12

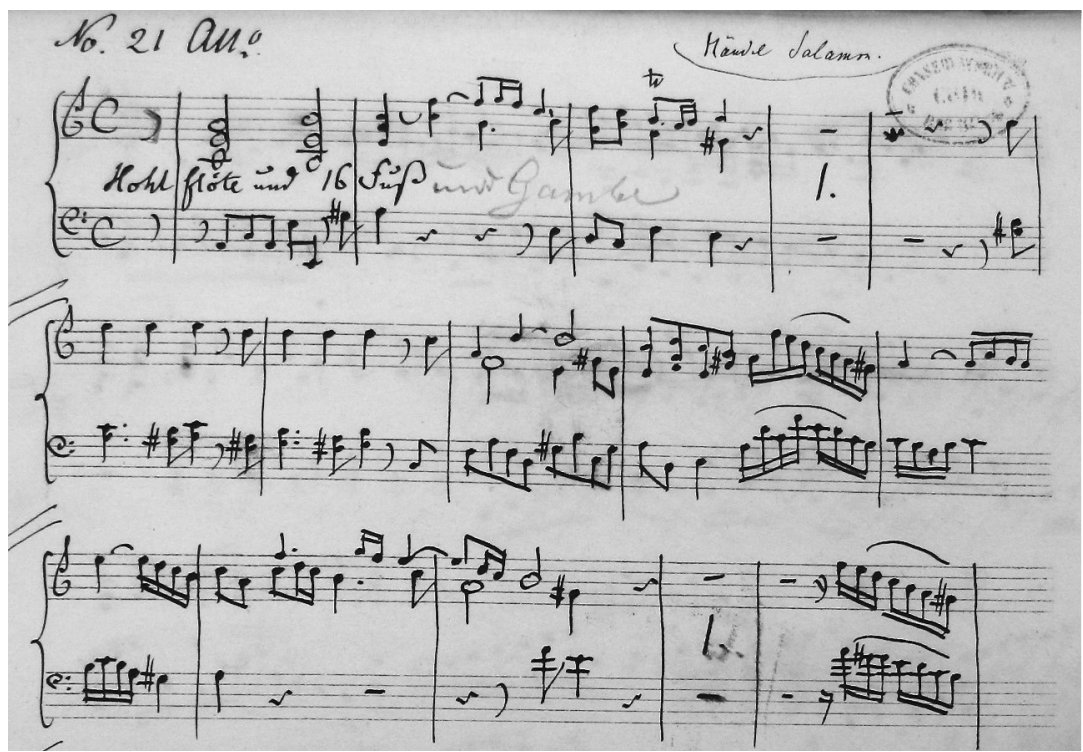

Abbildung 8b: D-KNh R 810, Arie „Thrice bless'd that wise“, T. 1-14

Im ersten Takt der Berliner Stimme strich Mendelssohn die noch erkennbaren Sechzehntel der rechten Hand, die sich in Sext- und Terzparallelen an der Violinstimme orientierten, und ersetzte sie durch eine Viertel $e^{\prime}-h^{\prime}$ und eine Viertelpause (vgl. Abbildung 8a). Diese Stelle änderte er abermals zugunsten von zwei gehaltenen Akkorden (vgl. Abbildung 8b). In T. 2 sollte die Orgelstimme ursprünglich mit dem Oktavunisono gehen, das sich zwischen Orchesterbass und Violinen findet (vgl. Abbildung 8a). Dieses Unisono erscheint in T. 11 zwischen Singstimme und Streichern erneut. Deshalb hat Mendelssohn in der Kölner Stimme die Führung der beiden Oberstimmen in T. 2 derjenigen von T. 11 angenähert (vgl. Abbildungen 8a mit 8b), ${ }^{59}$ um sie im weiteren Verlauf auch an anderen Parallelstellen

59 Diese Änderung ist in der Zwischenfassung noch nicht vorhanden. 
erneut aufzugreifen (z. B. in T. 16 und T. 63). ${ }^{60}$ Zudem lassen die beiden Sechzehntel der Oberstimme in T. 2 bzw. 11 (vgl. Abbildung 8b) die Sechzehntel des Streicherunisonos nachklingen. Weiterhin formt die Oberstimme in den ersten drei Takten einen Bogen aus, der sich ausgehend von $a$ ' hinaus über Händels Streichersatz bis zum $g$ " und wieder hinunter zum $d$ "le" spannt, um sich in T. 3 zugleich der Führung der ersten Violine anzuschließen (vgl. Abbildung 8b). Über die folgenden Pausen hinweg senkt er sich weiter hinab bis zum Ausgangston $a$ ' kurz vor Einsatz der Singstimme (T. 9). Somit wird das Händel'sche Orchesterritornell durch Mendelssohns Orgelstimme in einen großen Bogen gefasst. Durch Streichung der Figuration im vierten und fünften Takt zugunsten von Pausen (vgl. Abbildung $8 \mathrm{a}$ mit $8 \mathrm{~b}$ ) heben sich die Ritornellglieder stärker voneinander ab: Während der erste von der Orgel begleitete Abschnitt bis zum phrygischen Halbschluss reicht (T. 3), schweigt die Orgel im Folgenden (T. 4 f.), um mit der Sequenz ab T. 6 erneut einzusetzen (vgl. Abbildung 8b). Im Gegensatz zum Berliner Autograph bleibt konzertierende Sechzehntelfiguration bis zum Schluss des Ritornells aufgespart, um mit Einsatz der Singstimme (T. 9 f.) umso wirkungsvoller in Erscheinung zu treten. Händel greift den Ritornellabschnitt von T. 4 f. in T. 13 f. wieder auf, der an dieser Stelle die Singstimme unterbricht. Die Pause findet sich deshalb auch in T. 13 f., bevor bei Wiedereintritt der Singstimme (T. 14 f.) abermals Sechzehntel einfließen. Der weitere Verlauf zeigt, dass konzertante Figuration der Orgel vorrangig dann auftritt, wenn Singstimme oder Violinen nicht schon selbst in Sechzehnteln figurieren. Auch die lebhaften Sechzehntel, die im Berliner Autograph das Schlussritornell der Arie begleiten, sind Pausen und einer überwiegend schlichteren Figuration gewichen. Schließlich wurden die letzten fünf Takte mit Bleistift ganz gestrichen, so dass der Satz ohne Orgelbegleitung endet. ${ }^{61}$

Während der Proben dürfte sich herausgestellt haben, dass ein undurchsichtiges, diffuses Klangbild entsteht, sobald ein konzertantes Orgelsolo auf eine reich figurierte, stark besetzte Violinpartie trifft. Über weite Strecken führt solches Figurieren zwangsläufig zum Verwischen der Konturen und damit zur Verdunklung des formalen Aufbaus. Durch Reduzierung der Sechzehntel und gezielte Setzung von Pausen treten die Konturen des Satzes dagegen schärfer hervor. Diese Maßnahmen waren angesichts der Größe des Aufführungsapparates wohl notwendig, um der Klangmasse Transparenz zu verschaffen und eine Klarheit des Klangbildes zu erzielen.

Der Orgelpart für die übrigen Arien folgt weitgehend dem Berliner Autograph, doch finden sich in der Kölner Stimme wesentlich differenziertere Anweisungen zur Registrierung. Beispielsweise eröffnet das Anfangsritornell der Arie „Sacred raptures cheer my breast“ mit „Praestant 8 und Hohlfloete“, bevor mit Einsatz der Singstimme „blos Hohlfloete“ vorgeschrieben ist. In „Can I see my infant gor'd“ ist die Abregistrierung von „Viola

60 Vgl. auch die ähnliche Beobachtung zu Mendelssohns Orgelpart für das Duett „The Lord is my strength“ aus Israel in Egypt bei Moriz Violin, Über das sogenannte Continuo. Ein Beitrag zur Lösung des Problems, Wien [1911?], S. 23: „Er [Mendelssohn] kreiert fürs Ritornell eine neue eigene Melodie, die er dann aber freilich auch allezeit an den übrigen Parallelstellen, und zwar neben den Originalstimmen Händels verwendet.“ Zu Violins Studie vgl. Hellmut Federhofer, „Zu Felix Mendelssohns Händel-Interpretation im Urteil der Mit- und Nachwelt“", in: Musicologica Austriaca 8 (1988), S. 27-39.

61 Da Verkenius in seiner Partiturabschrift der Zwischenfassung der Kölner Stimme folgt, vgl. Anm. 72, konnte er diese Bleistiftstreichung nicht berücksichtigen. Das bedeutet jedoch nicht, dass sie deshalb nicht auf Mendelssohn zurückgehen kann, denn Verkenius hat eine vergleichbare Streichung am Ende der Arie „Praise ye the Lord for all his mercies past“ in seine Abschrift übernommen. Für die letzten Takte der Chöre „Music spread thy voice around“ und „Thus rolling surges rise“ ergänzte Mendelssohn die Anweisung „senza Organo“. 
da Gamba und Hohlf[öte]“ zu „Hohlf[öte] allein“ an den ersten Tempowechsel geknüpft (T. 1 ff: „Largo“, T. 36/37 ff.: „piu Allegro“). Die Tempobezeichnung „piu Allegro“ dürfte auf Mendelssohn zurückgehen, da sie sich weder in der Arnold-Ausgabe noch im SimrockKlavierauszug findet, die stattdessen "resoluto“ angeben. ${ }^{62}$ Besondere Aufmerksamkeit richtete Mendelssohn auch auf Tempoänderungen vor und nach Fermaten. ${ }^{63}$

Die Analyse zeigt die vielfältigen Mittel, mit denen Mendelssohn versucht, die Orgel als eigenständiges Instrument des Orchesters zu etablieren. Da er nur an drei Tagen die Möglichkeit hatte, den Salomon gemeinsam mit allen Mitwirkenden zu proben, scheint es nicht verwunderlich, dass er nach der ersten gemeinsamen Probe am 1. Juni, die trotz aller zuvor bereits geleisteten Arbeit missglückte, ${ }^{64}$,die ganze Idee und somit das ganze Fest für verrechnet ansah." Dass er dennoch kurz vor der Aufführung „Oberwasser“ gewann und das Projekt schließlich ein großer Erfolg für ihn wurde, ist nicht zuletzt auf die Kölner Orgelstimme zurückzuführen. Im Gegensatz zum Berliner Autograph zielt diese weniger auf konzertantes Figurieren, als vielmehr auf Vereinfachung, Vereinheitlichung, Klarheit, Kontrast, Schlagkraft und Schlusswirkung. ${ }^{65}$ Diese Tendenzen stehen mit seinerzeit favorisierten Klangvorstellungen in Zusammenhang, zeigen aber auch Mendelssohns Bemühen, der Klangmasse des großen Aufführungsapparates die nötige Transparenz zu verschaffen, ohne die eine Feinarbeit am Detail kaum möglich ist: „Es war [...] eine Freude, in den Proben die sichere Meisterhand zu beobachten, mit welcher Mendelssohn die feinste, zierlichste Arbeit nach und nach aus dem Groben herausschuf, - daneben aber auch die Aufmerksamkeit und Sorgfalt, mit welcher jeder Wink des Directors vom Orchester aufgenommen und befolgt wurde." 66

\section{Partitur}

Der erste Band von Verkenius' Partiturabschrift enthält den ersten Teil des Oratoriums und besteht aus 164 nummerierten Seiten im Querformat mit jeweils 16 Systemen. Der zweite Band umfasst auf 336 Seiten im Hochformat mit jeweils 20 Systemen die beiden übrigen Teile. Die Rückentitel beider Einbände weisen in goldfarbener Prägung folgenden Text auf: „Salomon. | Von | Händel | und | Mendelssohn. | Theil 1." bzw. „Theil 2. 3“. Auf dem

62 Für eine Reihe weiterer Tempoangaben lässt sich Ähnliches feststellen: Die Angabe „A Tempo Giusto“ für das Terzett „Words are weak“ findet sich nicht in den Orgelstimmen, die stattdessen „Allegro molto“ angeben (vgl. Abbildungen 6a und 6b). Auch Bezeichnungen wie „Larghetto“ oder „Andante Larghetto" der Arnold-Ausgabe sind in den Orgelstimmen gelegentlich zu „Andante con moto“, „Andante“ oder „,on moto“ geändert. Dies gilt z. B. für die Arien „Praise ye the Lord“, „Pious king, and virtuous queen" oder das Duett „Thrice bless'd be the king“.

63 Beispielsweise sollte im Duett „Thrice bless'd be the king“ ein Ritardando ursprünglich erst nach der Fermate (T. 138) einsetzen, bis mit Einsatz des Schlussritornells (T. 143 ff.) das Anfangstempo wieder aufgenommen wird („Tempo“). Bei der Umarbeitung der Stimme verbreiterte Mendelssohn das Ritardando stark, indem er es bereits vor der Fermate beginnen (ab T. 131), über sie hinausreichen und in ein „Adagio“ (T. 141) übergehen ließ. Während der Proben wurde dies erneut geändert: Das Ritardando mündet nun in die Fermate, und das ursprüngliche Tempo wird in direktem Anschluss und nicht erst mit Einsatz des Schlussritornells wieder aufgenommen.

64 Bis Ende Mai hatten bereits eine Reihe von Gesangs- und Instrumentalproben der für das Musikfest einzustudierenden Werke stattgefunden, die überwiegend von Domkapellmeister Carl Leibl, teilweise aber auch bereits von Mendelssohn selbst geleitet wurden, vgl. Niemöller, S. 62.

$65 \mathrm{Vgl}$. auch die ähnlichen Beobachtungen bei Hartinger, S. $303 \mathrm{ff}$.

66 Becher, ohne Seitenangabe. 
Titelblatt des ersten Bandes notierte Verkenius Hinweise auf einige Artikel verschiedener Musikzeitschriften, in denen Händels Oratorium besprochen wird. ${ }^{67}$ In beiden Bänden finden sich sowohl die zwei verschiedenen Besitzstempel von Verkenius, als auch die Stempel „Rheinische Musikschule Cöln“, „Conservatorium der Musik Cöln“ und „Bücherei der staatl. Hochschule für Musik Köln“. Somit fertigte Verkenius seine Abschrift, die nach seinem Tod (1841) in den Besitz seines Schwiegersohnes ${ }^{68}$ Ignaz Seydlitz gelangte, ${ }^{69}$ speziell für seine eigene Notenbibliothek an. Diese wurde „nach dem Tode seiner Tochter Sibylla Seydlitz im Jahre 1872 aufgrund einer testamentarischen Verfügung dem Konservatorium für Musik in Köln übergeben." ${ }^{\text {"70 }}$ Daher ist auf den Titelblättern beider Bände auch der Besitzvermerk "Seydlitz“" zu erkennen. Aus der bis heute bestehenden Rheinischen Musikschule (gegr. 1845) ging 1858 das erste Conservatorium der Musik in Cöln, die Staatliche Musikhochschule Köln und schließlich die heutige Hochschule für Musik und Tanz Köln hervor, ${ }^{71}$ deren Bibliothek beide Bände im Bestand der „Verkenius-Sammlung" verwahrt.

Verkenius' Abschrift enthält alle Sätze, die sich auch in der Druckausgabe Arnolds finden, und spiegelt Mendelssohns Ambitionen wider, Händels Oratorium mit Orgelbegleitung und ohne hinzugefügte Blasinstrumente aufzuführen, da sie fast die gesamte umgearbeitete Kölner Stimme berücksichtigt und die sonstige Instrumentation dem Werk Händels entspricht. ${ }^{72}$ Da die Umarbeitung der Kölner Stimme in der Nacht vom 1. zum 2. Juni 1835 erfolgte, kann die Abschrift des Notenteils erst nach diesem Datum vollendet worden sein. Dass diese Arbeit schon bald abgeschlossen gewesen sein dürfte, lässt sich aufgrund eines Briefs an Klingemann vom 6. Juli 1835 schließen, in dem Verkenius bittet, eine Partitur von Händels Occasional Oratorio für sich anzukaufen: „Sollte das Occasional nicht zu haben seyn, so wählen Sie mir ein anderes, nur nicht, Messias, Saul, Jephta - Josua Samson - Belsazar -Salomon [!] - Judas Maccabaeus und Deborah, weil ich diese schon besitze."73

Wie auch sonst für ihn typisch, hat Verkenius seine Kopie mit äußerster Sorgfalt erstellt: Zwischen den durchweg mit Lineal gezogenen Taktstrichen sind weitere, ebenfalls senkrechte Bleistiftstriche zu erkennen, um sicherzustellen, dass alle Noten derselben Zähl-

67 „S. Berliner musikalische Zeitung 1826. S. 152. / Leipziger allgem. musik. Zeit. 1826. S. 69 - 1831. S. 165. - 1833 S. 23. / Iris 1831. S. 53.“

68 Vgl. die Erläuterungen zum Brief Mendelssohns vom 14.08.1841 an Ignaz Seydlitz bei Max F. Schneider, Felix Mendelssohn Bartholdy. Denkmal in Wort und Bild, Basel 1947, S. 135 f.

69 Vgl. Art. „Das 39. niederrheinische Musikfest“, S. 188, rechte Spalte.

70 Fellinger, „Verkenius, Erich Heinrich“, S. 215.

71 Vgl. Dietmar von Capitaine, Conservatorium der Musik in Cöln. Zur Erinnerung an die wechselhafte Geschichte einer musikpädagogischen Einrichtung der Stadt Cöln, Norderstedt 2009.

72 Verkenius' Abschrift lässt den auf losem Bogen in Reinschrift notierten Orgelpart für die Arie „Thrice bless'd that wise discerning king " unberücksichtigt und folgt stattdessen der Zwischenfassung aus der Kölner Stimme. Vermutlich lag Verkenius der Bogen nicht vor, weil er wahrscheinlich erst später in die Stimme eingelegt wurde: Wohl um seine Zugehörigkeit zur Kölner Stimme zu markieren, erhielt er nicht nur eine eigene Stempelung „Conservatorium der Musik Cöln“, sondern auch den Vermerk „Händel Salomon.“ im Kopf der ersten Seite, vgl. Abbildung 8b.

73 D-B Autogr. I/261/2. Vermutlich kaufte Klingemann das Occasional Oratorio in der Ausgabe Arnolds an, denn ein solches Exemplar ist in einem Katalogband der Verkenius-Bibliothek gelistet, D-KNh L 348, Rubrik „Partituren“, Nr. 294. Der von Verkenius erstellte, insgesamt dreibändige Katalog führt außer Mendelssohns Einrichtung kein weiteres Exemplar des Salomon an. Der Band L 348, in dem Mendelssohns Einrichtung erwähnt ist, wurde von Verkenius bis kurz vor seinem Tod (1841) erstellt, denn die dort angegebenen Erscheinungsdaten der Ausgaben reichen bis ins Jahr 1840/41, vgl. ebd. z. B. Rubrik „Schriften über Musik“, Nr. 124-126. 
zeit auf gleicher Linie stehen. Im Gegensatz dazu fällt die nicht durchgehende, uneinheitliche Nummerierung der Einzelsätze in beiden Bänden auf. Die von Verkenius mit Tinte geschriebenen Nummern finden sich nur im ersten Band. Ab Salomons Rezitativ „Thou fair inhabitant of Nile" aus dem ersten Teil sind unterschiedliche Arten der Satzzählung mit Bleistift eingetragen worden. Dies wurde aber wohl frühestens 1862 vorgenommen, als Ferdinand Hiller Mendelssohns Einrichtung erneut aufführte. Bei den Vorbereitungen setzte sich Hiller insbesondere mit der „Numerirung“ der Sätze auseinander, die „besonderes Nachdenken [...] gemacht zu haben"74 scheint. Ähnliches dürfte bereits Verkenius beschäftigt haben, denn er wies den beiden Rezitativen „Vain are transient beauties" und "When thou art absent from my sight" die Nummer 15 und den beiden Arien „Indulge thy faith" und „With thee th'unshelter'd moor I'd tread" die Nummer 16 zu. Die dazwischenliegenden Sätze „My blooming fair" und „Haste to the cedar grove“ erhielten weder einen deutschen Text noch eine Nummerierung. Ein Vergleich mit dem bereits erwähnten Textbuch für das Musikfest 1835 ergab, dass alle Sätze von „Vain are transient beauties of the face" bis einschließlich „Haste to the cedar grove“ 1835 nicht musiziert wurden. Die Art dieser Nummerierung deutet darauf hin, dass sich Verkenius an der Zählung der Kölner Orgelstimme orientierte, aus der nicht eindeutig hervorgeht, welche Sätze mit der Angabe „No. 15 Recit No. 16 Aria tacet" gemeint sind.

Insgesamt strich Mendelssohn für die Kölner Aufführung 1835 folgende zehn Sätze: ${ }^{75}$

\author{
Erster Teil \\ Arie (Königin): „Bless'd the day when first my eyes“76 \\ Rezitativ (Zadok): „Vain are transient“ \\ Arie (Zadok): „Indulge thy faith“ \\ Rezitativ (Salomon): „My blooming fair“ \\ Arie (Salomon): „Haste to the cedar grove“ \\ Zweiter Teil \\ Rezitativ (Salomon): „Prais'd be the Lord“ \\ Arie (Salomon): „When the sun o'er yonder hills“ \\ Dritter Teil ${ }^{77}$ \\ Arie (Königin): „Ev’ry sight these eyes behold“ \\ Rezitativ (Salomon): „Gold now is common“ \\ Arie (Salomon): „How green our fertile pastures look“
}

Dieses Ergebnis wird durch die Kürzungsangaben bestätigt, die Mendelssohn in einem Brief an Klingemann vom 15. August 1832 macht, denn alle 1835 vorgenommenen Strei-

74 Vgl. Reinhold Sietz, Aus Ferdinand Hillers Briefwechsel 2 (= Beiträge zur rheinischen Musikgeschichte 48), Köln 1961, S. 14. Verkenius' ursprüngliche Nummerierung des Rezitativs Nr. 13 wurde mit Bleistift gestrichen und durch „11“ ersetzt.

75 Ermittelt durch Vergleich von Textbuch, Partitur und Orgelstimmen.

76 Das Berliner Autograph vermerkt, dass nach Salomos Arie „What though I trace“ (Nr. 10) ein nicht näher bezeichnetes Rezitativ folgen soll, bevor sich das Duett „Welcome as the dawn of day“ (Nr. 14) anschließt. Der Kopist der Kölner Stimme deutete Mendelssohns Angaben wie folgt: „No 11. und 12. bleibt weg. | No. 13 Recit tacet." Ein Vergleich von Textdruck und Partiturabschrift zeigt jedoch, dass Mendelssohn die beiden Rezitative „And see my Queen“ (Nr. 11) und „Thou fair inhabitant of Nile“ (Nr. 13) zu einem einzigen zusammenschloss und die dazwischenliegende Arie der Königin „Bless'd the day when first my eyes“ (Nr. 12) fortließ. Die Textunterlegung für diese Arie wurde erst in späterer Zeit in Verkenius' Abschrift eingetragen.

77 Die Sinfonia zu Beginn des dritten Teils ist zwar in Verkenius' Abschrift enthalten, doch findet sich weder in den Orgelstimmen noch im Textdruck ein Hinweis auf diesen Satz: Wie im gedruckten Kla- 
chungen werden dort bereits erwähnt. ${ }^{78}$ Allerdings beabsichtigte Mendelssohn 1832, noch weit mehr Sätze zu entfernen:

\section{Erster Teil}

Arie (Salomon): „What though I trace each herb“

Zweiter Teil ${ }^{79}$

Rezitativ (Levite): „Great prince, thy resolution's just“

Arie (Levite): „Thrice bless'd that wise discerning king“

Rezitativ (Zadok): „From morn to eve“

Arie (Zadok): „See the tall palm“

Der Schlusschor des zweiten Teils sollte gestrichen und durch den Chor „Praise the Lord“ aus dem dritten Teil ersetzt werden. ${ }^{80}$

\section{Dritter Teil}

Rezitativ (Königin von Saba): „Thy harmony’s divine“ - ab T. 14 gestrichen

Arie (Levite): „Pious king, and virtuous queen“

Rezitativ (Zadok): „Thrice happy king“

Arie (Zadok): „Golden columns, fair and bright“

Der Chor „Praise the Lord“ sollte an den Schluss des zweiten Teils gesetzt werden.

Dass Mendelssohn von diesen Streichungen und Modifikationen wieder abrückte, kann als Bemühen gewertet werden, Satzumstellungen zu vermeiden und mehr Sätze vom „ursprünglichen“"Händel aufzuführen, als 1832 geplant. 1835 gehörten Kürzungen sowohl zu Mendelssohns Bild vom „echten Händel“ als auch zu seinem aufführungspraktischen Konzept: Saul „ist gewiß eins der ergreifendsten und effectreichsten Händel'schen Oratorien, und so reich daß man nur etwa einiges wegzulassen, nicht zu bearbeiten und zuzufügen brauchte - also den ächten Händel hätte. Auch Salomon ist prächtig, und wohl noch nicht gehört." 81

Wie bereits erwähnt nennt Verkenius im Katalog seiner Bibliothek „C Klingemann“ als Autor der deutschen Übersetzung. Für die Aufführung 1835 war allerdings ein anderer deutscher Text vorhanden gewesen, den Mendelssohn jedoch vehement ablehnte. ${ }^{82}$ Daher sandte Klingemann ihm seine Übertragung, die auf eine frühere Fassung (1832) für eine

vierauszug fahren sowohl die Berliner als auch die Kölner Stimme nach dem Chor „Swell the full chorus“ (Nr. 33) direkt mit dem Rezitativ „From Arabia’s spicy shores“ (Nr. 34) fort. Dagegen ist der Instrumentalsatz („Allegro“), der auf die Ouvertüre folgt, in beiden Orgelstimmen vermerkt: „No 1 $1 / 2$ tacet. "Es ist indes möglich, dass ein entsprechender Vermerk für die Sinfonia nicht eigens notiert wurde. Bei der Wiederaufführung 1862 unter Hiller wurde der Satz musiziert, vgl. das Programmheft „39. Niederrheinisches Musik-Fest, gefeiert zu Cöln“, Köln [1862], D-DÜl K.W. 424 [Heft 39], S. 30: „Dritte Abtheilung. Instrumental-Einleitung“.

78 Vgl. Mendelssohn, Sämtliche Briefe, Bd. 3, hrsg. von Uta Wald und Juliane Baumgart-Streibert, Kassel u. a. 2010, S. 33 f. Mendelssohn plante zu diesem Zeitpunkt eine Aufführung des Oratoriums mit der Berliner Singakademie, die jedoch nicht zustandekam.

79 Laut Mendelssohns Ausführungen sollte direkt nach dem Anfangschor des zweiten Teils das Rezitativ folgen, „welches das Terzett mit den beiden Frauen einleitet“, ebd. S. 34. Gemeint ist wohl nicht erst der Abschnitt ab „Thou son of David“, wie im Kommentar zur Briefausgabe angegeben, sondern vielmehr das gesamte Rezitativ ab „My sovereign liege“, vgl. ebd. S. 493, Kommentar zu Zeile 42. In der Arnold-Ausgabe umfasst dieses Rezitativ beide Abschnitte.

80 Dies geht auch aus Mendelssohns Auflistung der Oratoriensätze hervor.

81 Brief vom 13.02.1835 an Verkenius, in: Mendelssohn, Sämtliche Briefe, Bd. 4, S. 169.

82 Vgl. Brief vom 07.04.1835 an Klingemann, in: ebd., S. 216. 
geplante, aber gescheiterte Aufführung mit der Berliner Singakademie zurückging, ${ }^{83}$ nochmals zu, ${ }^{84}$ doch konnten die Chorstimmen nicht mehr mit Klingemanns Text versehen werden. ${ }^{85}$ Ein Vergleich mit dem Programmheft 1835 ergab, dass die Chöre nach der Übersetzung gesungen wurden, die dem 1831 bei Simrock in Bonn erschienenen Klavierauszug bzw. den ebenfalls dort gedruckten Stimmen entstammt. Rund ein halbes Jahr nach der Aufführung des Salomon ließ sich Verkenius von Mendelssohn die gesamte Übersetzung Klingemanns zusenden, ${ }^{86}$ wohl um sie noch in seine Abschrift übertragen zu können. Somit ist es nicht verwunderlich, dass die Chöre im Programmheft 1835 eine andere Übersetzung aufweisen als in Verkenius' Partitur. Dass es sich bei den Chortexten seiner Abschrift tatsächlich um Texte Klingemanns handelt, wird aus einem Brief Mendelssohns an Verkenius deutlich: „Die Chöre habe ich Ihnen sämmtlich abgeschrieben, und dabei von neuem [bedauert,] daß wir sie nicht mit dem verbesserten Texte damals machen konnten; so [gewinnt] z. B. der herrliche vorletzte Chor [„Praise the Lord“] durch die dreimal wiederholten

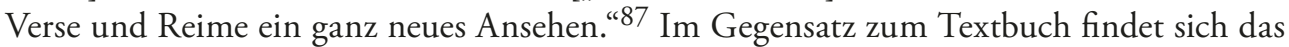
von Mendelssohn beschriebene Reimschema in der von Verkenius kopierten Übersetzung dieses Chores. ${ }^{88}$ Mendelssohn erwähnt an anderer Stelle, dass Klingemann ihm das ganze Oratorium „in Versen und Reimen, wie das Original“ 89 übersetzt habe und alle Rezitative und Arien nach dessen Übersetzung gesungen wurden. ${ }^{90}$ Da nur die Rezitative und Arien im Textbuch überwiegend gereimte Verse aufweisen und im Wesentlichen mit Verkenius' Partiturabschrift übereinstimmen, dürfte erwiesen sein, dass die deutschen Texte auch zu diesen Sätzen von Klingemann stammen und Verkenius Angabe „C Klingemann“ somit richtig ist.

Die Übersetzung, die Hiller für die Aufführung 1862 verwendete, entspricht größtenteils dem Text Klingemanns: ${ }^{11}$ Bis auf die Übersetzung des Chores „From the east unto the west" stimmen die im Programmheft abgedruckten Gesangstexte zum Salomon im Wesentlichen mit dem deutschen Text überein, der sich in Verkenius' Partiturabschrift findet. Es ist auch möglich, dass Hiller noch Zugang zum Klavierauszug hatte, in den Mendelssohn 1835 die Übersetzung Klingemanns eintrug. Dieses Exemplar, das einst zum Notenbestand des Singvereins Köln gehörte, ${ }^{92}$ befand sich 1909 in Kölner Privatbesitz. ${ }^{93}$

83 Vgl. Briefe vom 15.08. und 05.12.1832 an Klingemann, in: Mendelssohn, Sämtliche Briefe, Bd. 3, S. 33 f. und S. 81, sowie Brief vom 07.04.1835 an Klingemann, in: Mendelssohn, Sämtliche Briefe, Bd. 4, S. $215 \mathrm{f}$.

84 Brief vom 14.05.1835 an Klingemann, in: ebd., S. $237 f$.

85 Vgl. Niemöller, S. 51, insbesondere Fußnote 26.

86 Vgl. Brief vom 21.01.1836 an Verkenius, in: Mendelssohn, Sämtliche Briefe, Bd. 4, S. 377 f.

87 Ebd.

88 „Lobt den Herr(e)n jung und alt, | ihr Harfen schallt | seine Güt’ und Allgewalt | lobt den Herrn voll Majestät, | lobt ihn frühe lobt ihn spät, | deßen Name nie vergeht. | Jauchzt H o s a n n a mit Gesang | Durch die Lüfte dring der Klang | Gott allein sey Ehr und Dank."

89 Brief vom 04.05.1835 an Fanny Hensel, in: Mendelssohn, Sämtliche Briefe, Bd. 4, S. 233.

90 Vgl. Brief vom 14.05.1835 an Klingemann, in: ebd., S. 238.

91 Vgl. L. Bischoff, Vorwort“ zum Programmheft „39. Niederrheinisches Musik-Fest, gefeiert zu Cöln“, S. 12.

92 Vgl. Brief vom 21.01.1836 an Verkenius, in: Mendelssohn, Sämtliche Briefe, Bd. 4, S. 377.

93 Vgl. Ernst Wolff, „Briefe von Felix Mendelssohn Bartholdy an Kölner Freunde“, in: Rheinische Musikund Theater-Zeitung 10 (1909), S. 122, Fußnote 33. Da sich die Nummerierung der Orgelstimmen und die Übersetzung der Chöre im Textbuch 1835 im Wesentlichen am Simrock-Klavierauszug orientiert, wäre nach einem Exemplar dieser Ausgabe zu suchen, das Mendelssohns Textunterlegung enthält. 
In einigen Oratoriensätzen hat Verkenius den kompletten deutschen Text oder einzelne Textzeilen für die Vokalstimmen nicht unterlegt. Zum Teil wurde dies in späterer Zeit nachgetragen. Im ersten Teil gilt dies für die gesamte Arie „Heil dem Tag, wo ich erblickt“ („Bless'd the day when first my eyes“), im zweiten für die Zeile „Heil, dreifach, dreifach Heil des grossen Davids Sohn“ („Live, live for ever, pious David’s son“) aus dem Anfangschor und für den Schluss des nachfolgenden Rezitativs „Dank dir, o Gott“ („Prais'd be the Lord“). Das Rezitativ „My blooming fair“ und die sich anschließende Arie „Haste to the cedar grove“ aus dem ersten Teil blieben untextiert. Verkenius trug den deutschen Text wohl deshalb nicht ein, weil die genannten Sätze bis auf den Anfangschor des zweiten Teils bei Mendelssohns Aufführung 1835 gestrichen wurden.

Für die meisten Rezitative hatte Mendelssohn sowohl in der Berliner als auch in der Kölner Stimme keine Orgelbegleitung vorgesehen („tacet“-Vermerke). Verkenius ließ in manchen dieser Sätze ein oder zwei Systeme frei, in die Ferdinand Hiller gelegentlich eine schlichte akkordisch-homophone Begleitung eintrug, ${ }^{44}$ die überwiegend einem Streicherensemble zugewiesen ist und vermutlich in Zusammenhang mit seinen Aufführungsvorbereitungen 1862 steht. Über die Ausführung der Rezitative berichtet Heinrich Dorn 1844: „Bei den grossen niederrheinischen Musikfesten wurden die Recitative, wo sie nicht von Händel ausdrücklich für das volle Quartett gesetzt waren, von einem Contrabass und zwei Violoncells in meist vierstimmig gehaltenen Accorden begleitet. Diesen Arrangements unterzieht sich schon seit zwölf Jahren der tüchtige Violoncellist Bernhard Breuer ${ }^{95}$ in Cöln. “96

Im Vergleich zum Händel'schen Original weist Verkenius' Partiturabschrift einen modifizierten Blechbläserpart auf: So sind in der ersten Trompete alle Töne geändert, die über $a$ " hinausreichen. Zudem wurden oft Tiefoktavierungen vorgenommen, um die Ausführbarkeit zu erleichtern: Dies ist beispielsweise beim ersten Horneinsatz ab erneutem Eintritt des Cantus firmus im Chor „Praise the Lord“ (T. 97 ff.) der Fall. Ein anderer hoher Horneinsatz im Chor „From the censer curling rise“ (T. 76 f.) wurde gänzlich weggelassen.

Da Mendelssohns Händelaufführungen in der Regel eine weitreichende Auseinandersetzung mit den ihm zur Verfügung stehenden Notenmaterialien vorausging, stellt sich die Frage, mit welcher Genauigkeit die Partiturabschrift Resultate dieses oft sehr komplexen Prozesses widerspiegelt. Die Beantwortung dieser Frage bereitet Schwierigkeiten, da außer den Orgelstimmen bislang keine weiteren Notenmaterialien überliefert sind, die in direkter Beziehung zu Mendelssohns Aufführung des Salomon stehen und einen Vergleich mit Verkenius' Partitur ermöglichen könnten. ${ }^{97}$ Da sich die Seitenangaben sowohl im Berliner Autograph als auch in der Auflistung der Oratoriensätze auf die Partiturausgabe Arnolds beziehen, kann man davon ausgehen, dass Mendelssohn über ein solches Exemplar verfügte

94 Dies ergab ein Schriftvergleich mit autographen Partituren aus Hillers Nachlass, D-F. Zahlreiche teils mit Bleistift, teils mit Tinte geschriebene Vortragsbezeichnungen, die sich von Verkenius' Handschrift oft gut unterscheiden lassen, wurden wohl ebenfalls von Hiller eingetragen.

95 Breuer wurde u. a. als Violoncello-Lehrer des jungen Jacques Offenbach bekannt, vgl. Wulf Arlt, Art. „Breuer, Bernhard Joseph“, in: Rheinische Musiker 2, hrsg. von Karl Gustav Fellerer (= Beiträge zur rheinischen Musikgeschichte 53), Köln 1962, S. 11.

96 Heinrich Dorn, „Original oder Bearbeitung“, in: $A m Z 33$ (1844), Sp. 548. Für die Aufführung von Händels Josua unter Mendelssohns Leitung beim Kölner Musikfest 1838 sollte Breuer ein derartiges Arrangement mithilfe eines Klavierauszugs erstellen, vgl. Mendelssohns Brief vom 03.05.1838, mitgeteilt bei Esser, S. 54 f. Für Salomon lässt sich eine solche Einrichtung bislang nicht nachweisen, doch kann zumindest vermutet werden, dass sie vielleicht unter Verwendung des Simrock-Klavierauszuges angefertigt wurde.

97 Näheres zum Verbleib des Aufführungsmaterials findet sich bei Hartinger, S. $312 \mathrm{ff}$. 
oder es sich speziell für das Musikfest zu verschaffen gewusst hat. ${ }^{98}$ Dies dürfte Verkenius nicht entgangen sein, denn es war wohl seiner Initiative zu verdanken, dass das Comité des Niederrheinischen Musikfests alle 32 Bände der Arnold-Ausgabe kaufte, um sie Mendelssohn im Herbst 1835 als Geschenk zu übersenden. ${ }^{99}$ Bestimmte Unterschiede zwischen dieser Ausgabe und Verkenius' Abschrift könnten mit Änderungen, die Mendelssohn für die Aufführung 1835 vorgenommen haben dürfte, in Zusammenhang stehen. Sicherlich gilt dies für den modifizierten Blechbläserpart: Während Arnolds Partiturdruck Trompeten und Hörner in D verzeichnet, sind diese in Verkenius' Abschrift nach C transponiert. Für die Aufführung von Händels Josua beim Musikfest 1838 in Köln notierte Mendelssohn auf losem Bogen einen vereinfachten, nach $\mathrm{C}$ transponierten Part für Trompeten und Hörner. ${ }^{100}$ Verkenius' Abschrift des Blechbläserparts könnte auf eine entsprechende Vorlage, die vermutlich auch für Salomon existiert hat, zurückgehen.

Für das Musikfest stand ein Stimmmaterial, das Carl Friedrich Rungenhagen wohl für seine eigene Aufführung des Salomon 1832 bearbeitet hatte, zur Verfügung. Mendelssohn dürfte dieses Material mit der Ausgabe Arnolds verglichen und redigiert haben: „Gestern sind auch die sämmtlichen Salomo Stimmen von der Sing-Akademie gekommen; sie sind aber nicht wie Mutter sagt gemoselt, sondern die Veränderungen beschränken sich auf eine Baßposaune, ein Paar Hoboen und Hörner die Rungenhagen dazu geschrieben hat, und die wir nun wieder davon schreiben müssen." ${ }^{101}$ Die Chorstimmen aus diesem Bestand konnten seinerzeit nicht verwendet werden, „weil sie im Sopran-, Alt- und Tenorschlüssel geschrieben “102 waren. Um den Bedürfnissen der Sänger entgegenzukommen, schlug man vor, die gedruckten Stimmen in „moderner“ Schlüsselung bei Simrock zu bestellen. ${ }^{103} \mathrm{Im}$ Gegensatz zur Arnold-Ausgabe stehen die Sopran- und Altpartien in Verkenius' Abschrift im Violinschlüssel. Auch die Viola II ist nicht wie in Arnolds Partitur im Tenor-, sondern im Altschlüssel notiert.

Diese besonderen Merkmale zeigen, dass Teile der Abschrift sowohl bestimmte Modifikationen Mendelssohns berücksichtigen als auch in engem Zusammenhang mit den Bedürfnissen des damaligen Musikfestensembles stehen. Verkenius' Bemühen um Klingemanns Übersetzung und die Übertragung fast der gesamten Kölner Orgelstimme in seine Partitur verdeutlichen seine Absicht, Mendelssohns Salomon-Einrichtung wiederzuge-

98 Bevor sich das Comité endgültig für Salomon entschied, hatte sich Mendelssohn bei Verkenius erkundigt, ob die Oratorien Belsazar, Salomon und Saul in Köln vorhanden wären und um deren Übersendung nach Düsseldorf gebeten, vgl. Brief vom 25.02.1835 an Verkenius, in: Mendelssohn, Sämtliche Briefe, Bd. 4, S. 175. Zu bislang im Besitz Mendelssohns nachgewiesenen Drucken und Handschriften von Werken Georg Friedrich Händels, vgl. Wehner, „Zu Mendelssohns Kenntnis“, S. 193 ff.; zu Solomon vgl. ebd. S. $198 \mathrm{f}$.

99 Vgl. Niemöller, S. 63 f. und Mendelssohns Dankesbrief an Verkenius vom 05.10.1835, in: Mendelssohn, Sämtliche Briefe, Bd. 4, S. 313.

100 Vgl. Wehner, „Mendelssohn and the Performance“, S. 159-161.

101 Brief vom 31. März 1835 an Rebecka Lejeune Dirichlet, in: Mendelssohn, Sämtliche Briefe, Bd. 4, S. 209.

102 Niemöller, S. 51.

103 Vgl. D-KNmi, Archiv für Rheinische Musikgeschichte, A/I/21/1.24. 
ben. ${ }^{104}$ Indes verzeichnet die Partitur weder die 1835 vorgenommenen Satzstreichungen ${ }^{105}$ noch die vermutlich für Kontrabass und Violoncelli eingerichtete Rezitativbegleitung. Einzeichnungen, die auf eine Concertino-Ripieno-Dynamik hindeuten, stammen aus späterer Zeit. ${ }^{106}$ Die letzte Fassung des Orgelparts für die Arie „Thrice bless'd that wise discerning king" konnte Verkenius ebenfalls nicht berücksichtigen, weil ihm der lose Bogen, auf dem er notiert ist, zum Zeitpunkt der Erstellung seiner Partitur wohl nicht zugänglich war. ${ }^{107}$ Auch geänderte Tempobezeichnungen, die wohl auf Mendelssohn zurückzuführen sind, finden sich nicht in Verkenius' Abschrift, die stattdessen der Arnold-Ausgabe folgt.

Für seine Händel-Aufführungen ergänzte Mendelssohn in der Regel Angaben zur Dynamik und Artikulation. In welchem Umfang dies auch für Salomon 1835 der Fall war, kann allein anhand der Partiturabschrift nicht entschieden werden, da eine Vielzahl der Vortragsangaben erst später von Ferdinand Hiller ergänzt wurde und diejenigen, die mit bloßem Auge sicher Verkenius' Hand zugewiesen werden können, weitgehend mit der Druckausgabe Arnolds übereinstimmen. Weiterführende Erkenntnisse zu diesem Aspekt könnten indes durch künftige Spezialuntersuchungen gewonnen werden.

\section{Rezeption}

Mendelssohns Aufführung des Salomon wirkte in den folgenden Jahrzehnten in besonderer Weise nach: Unter den Zuhörern der Aufführung, die Hiller 1862 leitete, befand sich auch der junge Johannes Brahms. ${ }^{108}$ Dieser war so beeindruckt von Mendelssohns Arbeit, dass er über zehn Jahre später an Hiller schrieb: „Darf ich Ihnen mit einer recht dringenden Bitte kommen? Sie besitzen in Köln eine Bearbeitung des Händelschen Salomo, ich glaube gar von Mendelssohn. Darf ich mir diese, wenn Sie wollen auf kürzeste Zeit, zur Ansicht ausbitten? [...] können Sie Ihrem Archivar wohl die Ordre geben mir jenen Salomo zu schicken? Es kommt mir darauf an die Partitur recht bald zu haben [...]". ${ }^{109}$ Hiller, der Mendelssohns Einrichtung beim fünften Gesellschafts-Conzert am 7. Dezember 1869 im Kölner Gürzenichsaal erneut aufgeführt hatte ${ }^{110}$ und seit 1858 Direktor des Conservatoriums der Musik in Coeln war, ${ }^{111}$ antwortete ihm bald darauf: „Die Partitur des Salomo mit der Mendelssohnschen Orgelstimme wird wohl heute noch an Sie abgehen. Da dieselbe eine Art von Kostbarkeit aus der Verkeniusschen Bibliothek [ist], so bedurfte es einiger kleiner Umstände. Daß Sie mit unserm Hauptbibliothekar, Herrn Justizrath Steinberger,

104 In bestimmter Hinsicht geht Verkenius über seine Angabe „so wie es 1835 beim Musikfest in Köln aufgeführt wurde" hinaus, da seine Abschrift die Chortexte Klingemanns verzeichnet, die, wie bereits erläutert, für die damalige Aufführung nicht berücksichtigt werden konnten. Die deutschen Texte einiger Rezitative und Arien, die Mendelssohn 1835 gestrichen hatte, übertrug er ebenfalls in seine Abschrift.

105 Mit Bleistift in Verkenius’ Partitur eingetragene Kürzungsvermerke wie „bleibt weg“ oder „vide“ stehen im Zusammenhang mit Hillers Aufführung 1862.

106 Beispielsweise sollten die ersten 10 Takte des Chores „From the censer" vom „Solo-Orchester" ausgeführt werden (,senza r[ipieno]“), bevor mit Einsatz der Vokalstimmen (T. 11) die Ripieno-Instrumente hinzutreten („,con r[ipieno]“).

107 Vgl. Anm. 72.

108 Vgl. Art. „Das 39. niederrheinische Musikfest“, S. 188, rechte Spalte.

109 Brief vom Juli 1873, in: Aus Ferdinand Hillers Briefwechsel 3, hrsg. von Reinhold Sietz (= Beiträge zur Rheinischen Musikgeschichte 56), Köln 1964, S. 114.

110 Vgl. ebd. S. 8 ff.

111 Vgl. von Capitaine, S. 25 ff. 
vor ein paar Monaten in Tutzing [?] so freundlich geplaudert, hat die Sache wesentlich erleichtert. Sie sehen, wozu Liebenswürdigkeit gut ist." 112 Nach Eingang von Verkenius' Partiturabschrift verlangte Brahms auch die teilautographe Kölner Stimme: „Es ist eine wahre Lust die Mendelssohnsche Arbeit zu sehen; hätten wir doch zu Bachschen Cantaten solche Orgelstimme! Nun sässe ich gern weiter m[it] Lust und auch m[it] Vortheil daran. Sie haben gewiß die ,ausgeschriebene Orgelstimmé. Dürfte ich mir diese für kurze Zeit ausbitten um sie in München copiren zu lassen? Dagegen kann dann die Partitur jeden Tag zurück gehen." ${ }^{113}$ Ein Vergleich mit der Kölner Stimme ergab, dass sich diese von Brahms in Auftrag gegebene Kopie im Archiv der Gesellschaft der Musikfreunde in Wien befindet. ${ }^{114}$ Inwiefern die Abschrift, die zahlreiche Eintragungen von Brahms' Hand enthält, in Zusammenhang mit der Aufführung von Händels Salomo steht, die unter Brahms' Leitung mit dem Singverein am 31. März 1874 in Wien stattfand, ${ }^{115}$ bleibt zu untersuchen.

Richard und Cosima Wagner hörten am 5. Dezember 1872 im großen Saal der Tonhalle Düsseldorf den ersten Teil des Oratoriums mit dem städtischen Musikverein unter Leitung von Julius Tausch, der für seine Aufführung Mendelssohns Orgelstimme verwendete. ${ }^{116}$ Cosima berichtet: „Um 4 Uhr fahren wir nach Deutz und von da nach Düsseldorf, wo uns gemeldet war, daß der ,Salomo' von Händel mit gastierenden Sängern gegeben würde. [...] Wir hören den ersten steifen, langweiligen Teil des Oratoriums an und eilen dann fort [...]." 117 In seiner Schrift „Einblick in das heutige deutsche Opernwesen“ (1873) spottete Richard Wagner: „[...] so erfährt man einmal etwas von einem ganz herrlichen, durchaus klassischen, Händel'schen ,Salomon', zu welchem der selige Mendelssohn selbst für die Engländer die Orgelbegleitung gesetzt hat. [...] Und herrliche Musiksäle bauen sie ihren hohen Priestern auf: darin sitzen sie, verziehen keine Miene, lesen im Texte nach, wenn oben auf dem Bretterbau ihre lieben Verwandten Jehova-Chöre singen, und Jupiter selbst ihnen den Takt dazu schlägt. Dergleichen erlebte ich zu Düsseldorf [...]."118

Im Rahmen der Niederrheinischen Musikfeste wurde Mendelssohns Einrichtung am 4. Juni 1876 in Aachen unter der Leitung des städtischen Musikdirektors Ferdinand Breu-

112 Brief ohne genaues Datum, „Anno 1873“, in: Aus Ferdinand Hillers Briefwechsel 3, S. 115.

113 Brief vom Juli 1873 , ebd. S. 115.

114 A-Wgm H 27944 (Händel III 9564).

115 Vgl. Imogen Fellinger, „Das Händel-Bild von Brahms“, in: Göttinger Händel-Beiträge 3 (1989), S. 235-257, hier: S. 237 ff. Den Schlusschor des ersten Teils „May no rash intruder“ dirigierte Brahms wenig später erneut, vgl. ebd. S. 237.

116 Vgl. Carl Friedrich Glasenapp, Das Leben Richard Wagners 5, Leipzig 1907, S. 48 und Programmheft „Allgemeiner Musik-Verein. [...] Drittes Concert unter Leitung des Königlichen Musik-Directors Herrn Julius Tausch“, 05.12.1872, D-Stadtarchiv Düsseldorf, G 72 (1872). Die vier autographierten Chorstimmen, die sich im Notenarchiv des Düsseldorfer Musikvereins befinden, weisen den Text auf, den Hiller 1862 verwendet hatte, vgl. D-DÜhh 161.2. Doch zeigt das Programmheft, dass man bei der Aufführung nicht Klingemanns Text, sondern der Übersetzung des Simrock-Klavierauszugs folgte, vgl. D-DÜhh 161.1 [vier Exemplare]. Bei der Aufführung 1872 benutzte man auch die ebenfalls bei Simrock erschienenen gedruckten Stimmen: In einer Tenor-Stimme, D-DÜhh 161.1, finden sich auf S. 19 handschriftliche Textänderungen im Chor „Swell the full chorus“: „o nennt ihn ihr Sänger den Stolz unsrer Zeit“ statt „o nennt ihn ihr Sänger zum Stolze unsrer Zeit“. Dieser modifizierte Text findet sich auch im Programmheft 1872.

117 Cosima Wagner, Die Tagebücher, Bd. 1, hrsg. von Martin Gregor-Dellin und Dietrich Mack, München 1976, S. 607.

118 Richard Wagner, Gesammelte Schriften und Dichtungen, Bd. 9, Leipzig 1898, S. 282 f. 
nung erneut gegeben: ${ }^{119}$ Die hier verwendete Orgel mit drei Manualen, Pedal, Posaune 16' und 32' wurde von Georg Stahlhut, Aachen (Burtscheid), erbaut, bestand aus insgesamt 43 Registern und ca. 2500 klingenden Pfeifen. ${ }^{120}$ Während der Text der Chöre überwiegend demjenigen Klingemanns entsprach, griff man für die Übersetzung der Rezitative und Arien auf die Ausgabe der Deutschen Händel-Gesellschaft zurück. ${ }^{121}$ Wie dieser Einblick in die Rezeptionsgeschichte zeigt, reichte die Wirkung, die von Mendelssohns Aufführung des Salomo 1835 ausging, bis weit in die zweite Hälfte des 19. Jahrhunderts. Selbst die Bezeichnung „nach der Original-Partitur“, 122 die mit diesem Ereignis verbunden war, ${ }^{123}$ taucht in allen genannten Programmheften der späteren Aufführungen unter Hiller, Tausch und Breunung wieder auf. Zudem folgte man stets allen 1835 von Mendelssohn vorgenommenen Streichungen. Bei der ersten Wiederaufführung 1862 ließ Hiller darüber hinaus noch einige weitere Sätze fort, unter denen sich auch die beiden Bass-Arien des Leviten „Thrice bless'd that wise discerning king“ und „Pious king, and virtuous queen“ befanden, ${ }^{124}$ für die Mendelssohn eine Orgelbegleitung geschrieben hatte. Die einzige Arie des zweiten Weibes wurde ebenfalls gestrichen. Hillers zusätzlichen Kürzungen folgten Tausch und, abgesehen von einer Ausnahme, auch Breunung. ${ }^{125}$ Dies zeigt, dass Ferdinand Hiller nicht nur zur Verbreitung der wieder aufgefundenen Quellen maßgeblich beitrug, sondern auch die Konzepte späterer Aufführungen beeinflusste.

119 Vgl. Programmheft „53. Niederrheinisches Musikfest, gefeiert zu Aachen, Pfingsten, 4., 5. und 6. Juni 1876“, D-DÜl K.W. 424 [Heft 53], S. 4 und S. 15 ff. An der Orgel saß „Concertmeister Winkelhaus aus Aachen", vgl. ebd. S. 58.

120 Vgl. ebd. S. 6 f.

121 Vgl. ebd. S. 11.

122 Offensichtlich meinte Mendelssohn damit die Arnold-Ausgabe.

123 Vgl. Niemöller, S. 51 f.

124 Dies lässt sich auch aus der bereits erwähnten Rezension der Aufführung schließen, in der die Arie des Leviten „Praise ye the Lord“ aus dem ersten Teil als „die einzige für Bass-Solo im ganzen Oratorium“ bezeichnet wird, vgl. Art. „Das 39. niederrheinische Musikfest“, S. 189, linke Spalte.

125 Im Gegensatz zu Hiller ließ Breunung das Rezitativ „When thou art absent“ und die zugehörige Arie „With thee th'unshelter'd moor I'd tread“ der Königin nicht fort. 